\title{
Mixing and reaction in turbulent plumes: the limits of slow and instantaneous chemical kinetics
}

\author{
N. Mingotti $\dagger$ and S. S. S. Cardoso \\ Department of Chemical Engineering and Biotechnology \\ University of Cambridge, Philippa Fawcett Drive, Cambridge CB3 0AS, UK
}

(Received xx; revised xx; accepted xx)

We investigate the behaviour of a reactive plume in the two limiting cases of slow and instantaneous chemical reactions. New laboratory measurements show that while the slow reaction between the source and entrained chemical species takes place within the whole volume of each eddy in the plume, the fast reaction develops preferentially at the periphery of the eddies. We develop a new model that quantifies the mixing of the reactive buoyant fluids at the Batchelor scale and thereby the progress of the fast reaction. We present a series of new experimental results which suggest that a critical distance from the source, $z_{\text {crit }}$, exists at which the volume of fluid which is entrained from the ambient is equal to that which is mixed within the plume at the Batchelor scale. For $z>z_{\text {crit }}$, only a fraction of the entrained fluid is rapidly mixed and reacts with the plume fluid. The results of the new experiments enable us to quantify the distance from the source at which an instantaneous reaction reaches completion, and show that it can be significantly larger than the distance $L_{s}$ at which the stoichiometric dilution of the plume fluid is achieved. In the limit of an instantaneous reaction, the longitudinal profiles of source chemical concentration in the plume depend on $\left(z_{\text {crit }} / L_{s}\right)^{5 / 6}$. The predictions of the model are validated against the experimental results, and the profiles of source chemical concentration in the plume for slow and fast reactions are compared.

Key words: Plume, mixing, reaction

\section{Introduction}

Turbulent plumes are observed in a variety of natural flows, such as the fluid columns that rise above hydrothermal vents in the deep ocean (Lupton et al. 1985) or volcanic vents during an eruption (Woods 2010; Sparks et al. 1997). At a range of different scales, turbulent plumes are also encountered in industrial flows, including discharges of smoke from chimneys, effluents from submerged pollutant outlets and leaks from pipelines (Campbell \& Cardoso 2010). While rising above a localised source of buoyancy, plumes entrain fluid from the surrounding ambient (Morton et al. 1956), leading to a progressive dilution of the source fluid. Internal processes such as chemical reaction, dissolution and phase change may also contribute to buoyancy changes in the plume.

A number of recent studies have considered the interaction of chemical reaction and the buoyant flow in turbulent plumes. Most of these have focussed on the effects of reaction

$\dagger$ Email address for correspondence: nm441@cam.ac.uk 
on buoyancy, through a change in composition or enthalpy, and its influence on the flow field. Conroy \& Llewellyn Smith (2008) and Campbell \& Cardoso (2010) investigated the behaviour of a single-phase plume with internal buoyancy generation. Their numerical and analytical studies describe the behaviour of the plume both in the region near the source and in the far field, and quantify the impacts of different buoyancy fluxes produced by the reaction relative to the flux at the plume source. In the context of carbon-dioxide and methane releases in the ocean, Cardoso \& McHugh (2010) and Domingos \& Cardoso (2013) considered a two-phase plume with a first-order reaction at the interface between the droplets/bubbles and the plume liquid. They identified the conditions for which the plume buoyancy is controlled by either the density stratification in the environment or by the chemical dissolution. Woods \& Caulfield (1992) noted that when a sufficiently large flux of negative buoyancy is generated during the non-linear mixing of the plume and ambient fluids, then the bulk density of the plume fluid may become larger than that of the the surrounding ambient fluid at a finite distance from the source. In this case the plume gradually transitions into a collapsing fountain. Woods \& Caulfield $(1992 ; 1995)$ explored some of the dynamics of this transition using experiments with mixtures of methanol and ethylene glycol (MEG) and water, which exhibit a reversal of buoyancy as the MEG mixes with water. They reported intermittent formation of collapsing fountains and convective plumes in a range of experimental conditions.

When the chemical interaction has negligible effects on the buoyancy of the plume, the dynamics of the flow are described by the classical theory (cf. Morton et al. 1956), while the kinetics of the reaction determine the process of consumption of reactants in the fluid. For example, when warm, acidic industrial discharges are released into the natural environment, buoyant plumes rise through and mix with the surrounding ambient fluid. Ulpre et al. (2013) presented the results of a series of laboratory experiments, in which an acidic plume descended through a tank containing an alkaline solution. They quantified the distance from the source at which the plume fluid reaches the stoichiometric dilution, and measured the longitudinal concentration of source chemical in the two cases of a strong acidic plume descending through either (i) a strong or (ii) a weak alkaline ambient.

In all the above studies, it was assumed that the chemical reaction occurs on a larger time scale than the small-scale mixing in the plume. Indeed, few studies have considered plume flows where the reaction is limited by turbulent mixing. Komori \& Ueda (1984) measured the concentration of chemical species in a gaseous plume undergoing a moderately fast, second-order chemical reaction and found that the turbulence effects on the reaction rate are significant: in particular, they noted that the effect of the concentration fluctuations on the reaction rate can be as much as 20 times larger than that of the mean concentrations. Domingos \& Cardoso (2015) demonstrated that turbulent thermals undergoing an instantaneous chemical reaction are non-uniformly mixed and quantified the delay in the completion of the reaction, compared to the classical wellmixed scenario.

In the present study, we address this gap in knowledge by investigating the behaviour of a plume with a second-order chemical reaction that does not affect the buoyancy flux of the plume. We present and contrast the results of a series of new laboratory experiments focussing on two limiting scenarios, those of fast and slow reactions compared to the mixing across the plume. We measure for the first time the size of eddies containing reactant and its evolution along the plume. We show that a fast reaction occurs mainly at the periphery of the turbulent eddies and we relate the local rate of chemical conversion to that of mixing on the Batchelor scale. We propose a new theoretical model to predict the length scale for complete reaction as a function of the small-scale mixing in the 

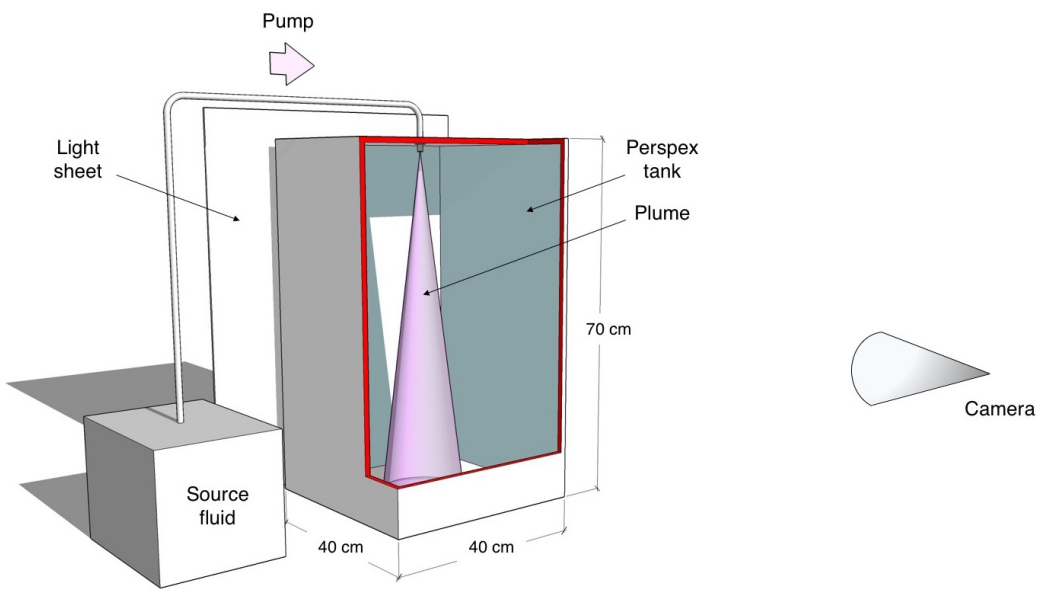

FIGURE 1. Schematic representation of the experimental setup.

plume. Our model is validated with the experimental results, and shows that in the limit of an instantaneous reaction, the distance from the source at which the source reactant in the plume is consumed can be significantly larger than that at which the stoichiometric dilution is achieved. This means that reactive discharges of buoyant fluid will be fully neutralised at larger distances from the source than previously reported. The experimental observations presented in the paper enable us to quantify these distances, as well as the longitudinal concentration profiles of source reactant in the plume.

The structure of the paper is as follows: in section 2, we describe the experiments and the technique used to process the data. In section 3, we describe the chemical reactions used in the experiments, and give details about their kinetics. In section 4, we discuss the main experimental observations and the different processes of consumption of reactants in plumes with a fast or a slow reaction. We present the new model in section 5 and draw conclusions in section 6 .

\section{Setup and calibration of experiments}

\subsection{Experimental setup}

A series of experiments was carried out to explore the behaviour of turbulent, singlephase plumes with a chemical reaction. The experiments were run in a perspex tank of dimensions $40 \times 40 \times 70 \mathrm{~cm}$ (figure 1). Before the beginning of each experiment, the tank was filled with a dilute aqueous solution of an acid. (i) Acetic, or (ii) nitric, or (iii) ascorbic acid were used to investigate the effects of different reactions (see table 1). To study the impacts of different source conditions, two round nozzles of a radius 0.6 and $1.2 \mathrm{~mm}$ were used in the experiments. During each experiment, one of the nozzles was placed at the top of the tank and was connected to a glass beaker containing an aqueous solution of (i) ammonium hydroxide, or (ii) sodium hydroxide, or (iii) methylene blue (see table 1). To increase the density of the source fluid in the beaker relative to that of the fluid in the tank, either sodium chloride or sucrose was added to the solution (see table 1). In experiments (i) and (ii), a few drops of phenolphthalein colour indicator were added to the clear fluid in the beaker (see table 1); the addition of the indicator did not affect the density or viscosity of the fluid. Owing to the presence of methylene blue in the solution, no extra dye was added to the fluid in the beaker in experiments (iii).

During each experiment, the relatively dense fluid in the beaker was supplied to the 


\begin{tabular}{ccccc} 
Exp. & Ambient fluid & Plume fluid & $\begin{array}{c}\text { Increase density } \\
\text { of plume fluid }\end{array}$ & Dye \\
\hline (i) & $\begin{array}{c}\mathrm{CH}_{3} \mathrm{COOH} \\
\text { acetic acid }\end{array}$ & $\begin{array}{c}\mathrm{NH}_{4} \mathrm{OH} \\
\text { ammonium hydroxide }\end{array}$ & $\begin{array}{c}\text { Sodium } \\
\text { chloride }\end{array}$ & Phenolphthalein \\
(ii) & $\begin{array}{c}\mathrm{HNO}_{3} \\
\text { nitric acid }\end{array}$ & $\begin{array}{c}\mathrm{NaOH} \\
\text { sodium hydroxide }\end{array}$ & $\begin{array}{c}\text { Sodium } \\
\text { chloride }\end{array}$ & Phenolphthalein \\
(iii) & $\begin{array}{c}\mathrm{H}_{2} \mathrm{~A} \\
\text { ascorbic acid }\end{array}$ & methylene blue & Sucrose &
\end{tabular}

TABLE 1. Reactive systems used in the experiments.

tank using a peristaltic pump at a controlled flow rate $Q_{0}$ in the range $1-2 \times 10^{-6} \mathrm{~m}^{3}$ $\mathrm{s}^{-1}$ (see tables 2-4). On entering the tank, the outflow from the nozzle rapidly became turbulent (see Appendix A), and formed a dense plume which descended through the surrounding ambient fluid. The tank was backlit using a light panel of dimensions $50 \times$ $80 \mathrm{~cm}$ (manufactured by Electro-LuminX Lighting Co.) positioned at the rear of the tank (see figure 1). A Nikon D300 camera, located in front of the tank, was used to capture pictures at a frequency of $5 \mathrm{~Hz}$ (figure 2a). Each image had 2136 pixels in the horizontal direction and 3212 pixels in the vertical direction, resulting in a resolution $0.218 \mathrm{~mm} /$ pixel. The experiments were performed in a dark room, and so the only light detected by the camera had passed through the fluid in the tank. Consequently, the line-of-sight average distribution of the dyed plume fluid in the tank could be inferred based on the distribution of the light attenuation in the images. In figure $2 \mathrm{~b}$, false colours are used to illustrate the instantaneous distribution of the dyed plume fluid 10s after the beginning of experiment 1 (see table 2 ). In order to ensure that the results are repeatable and reproducible, each reactive experiment was repeated up to three times. In figures $3,4,7,10$ and 11 we show the different results obtained when repeating experiment 5; these results are labelled "5a", "5b", and " $5 \mathrm{c} "$ ". For all other experiments, we plot the average of the results obtained from each run. For calibration purposes, and in order to compare the properties of a plume with chemical reaction to those of an inert plume, each reactive experiment was also repeated using fresh water as the ambient fluid in the tank. Each experiment typically lasted 2.5-3 minutes, during which approximately 750900 photographs were captured. A time average of these photographs was calculated for each experiment to estimate the average distribution of the light attenuation produced by the plume fluid during the experiment (figure 2c).

\subsection{Turbulent plumes in fresh water}

The outcomes of the experiments with no chemical reaction were analysed first, in order to: (i) estimate the errors associated with the light attenuation experimental technique; (ii) identify the virtual origin of the plume and estimate its entrainment coefficient; and (iii) measure the time-averaged light attenuation produced by the plume fluid as it becomes increasingly diluted while descending through the tank. This will be used as a reference in section 5 , in which the effects of the chemical reaction are investigated.

In a plume descending through an inert unstratified ambient, the buoyancy flux

$$
B=2 \pi \int_{0}^{\infty} \frac{\rho-\rho_{a}}{\rho_{a}} r g u d r
$$



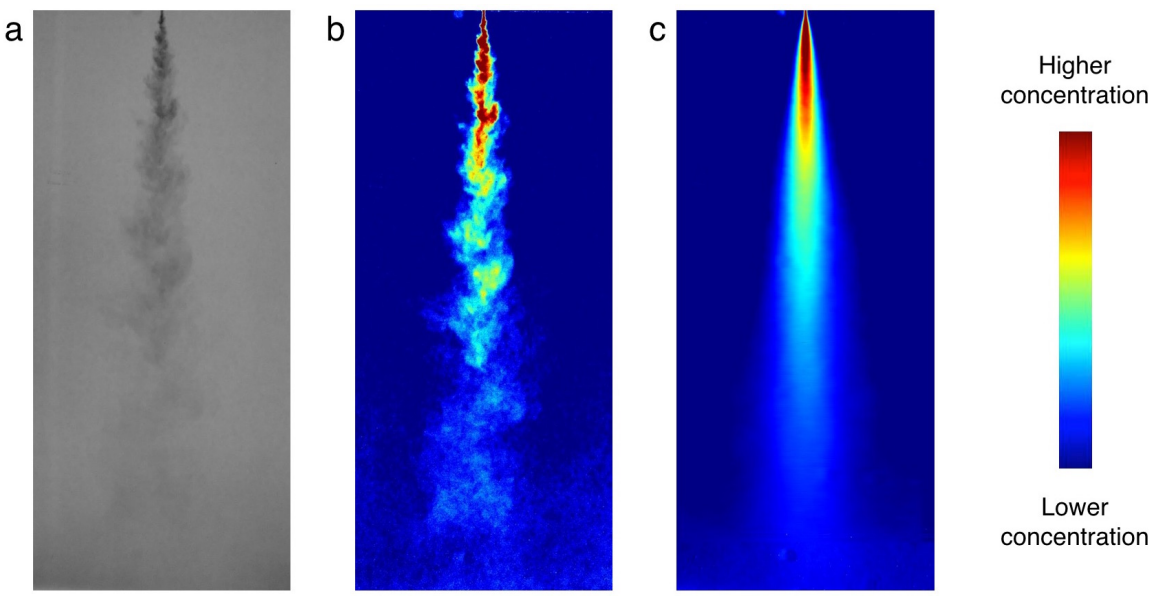

Figure 2. (a) Photograph of an inert plume descending through fresh water; (b) The depth-averaged distribution of light attenuation is visualised using false colours; (c) Time- and depth-averaged distribution of light attenuation. This image has been obtained by averaging 200 photographs which were captured during $40 \mathrm{~s}$ at the beginning of the experiment.

is conserved (Morton et al. 1956); here, $\rho$ and $\rho_{a}\left(\mathrm{~kg} \mathrm{~m}^{-3}\right)$ are the densities of the plume and the ambient fluids respectively, $r(\mathrm{~m})$ is the radial distance of a parcel of fluid from the plume centreline, $g\left(\mathrm{~m} \mathrm{~s}^{-2}\right)$ is the gravitational acceleration, and $u(\mathrm{~m}$ $\mathrm{s}^{-1}$ ) is the downward speed of the plume fluid. In order to estimate the errors associated with the image analysis technique, the photographs captured during each experiment with no chemical reaction were used to quantify the flux of light attenuation at different distances from the plume source, and verify whether this flux was conserved. Figure 3 illustrates how the flux of light attenuation was measured. For each picture captured during an experiment, we measured the horizontally averaged profile of light attenuation in a rectangular region surrounding the plume (red box in figure 3a). A time series of these profiles is plotted in figure 3b: dark fronts stretching diagonally are observed in this image, illustrating the downward motion of the plume eddies over time. We used the Hough transform as available in Matlab to identify the fronts (red lines in $3 \mathrm{~b}$ ), measure their gradients, and thereby estimate the speed of the eddies at different distances below the source (cf. Mingotti \& Woods 2016). We multiplied the cross-sectional light attenuation profiles by the associated speed profiles to infer the magnitude of the flux of light attenuation. Figure $3 \mathrm{c}$ shows that across a number of experiments the timeaveraged fluxes of light attenuation were approximately conserved, with fluctuations of order $5-7 \%$, in the region of the tank spanning between the plume source and the expected stoichiometric distance in the reactive experiments below (cf. Ulpre et al. 2013). This indicates that in our experiments the attenuation of the light through the tank was approximately proportional to the concentration of dye in the plume fluid in the tank.

Plume theory indicates that in the absence of any chemical reaction, the reduced gravity of the plume fluid $g^{\prime}$ and the plume radius $b$ vary with distance from the source, $z$ (Morton et al. 1956) according to

$$
g^{\prime}\left(z+z_{0}\right)=\frac{B}{Q\left(z+z_{0}\right)} \propto B^{\frac{2}{3}}\left(z+z_{0}\right)^{-\frac{5}{3}}
$$

and

$$
b\left(z+z_{0}\right)=\frac{6}{5} \alpha\left(z+z_{0}\right)
$$




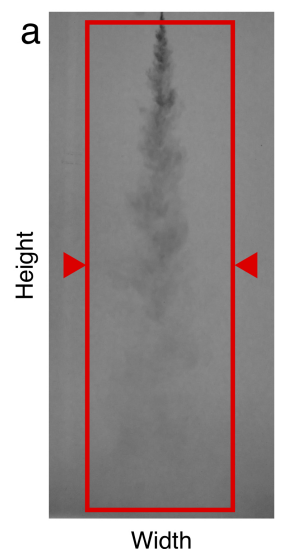

Width

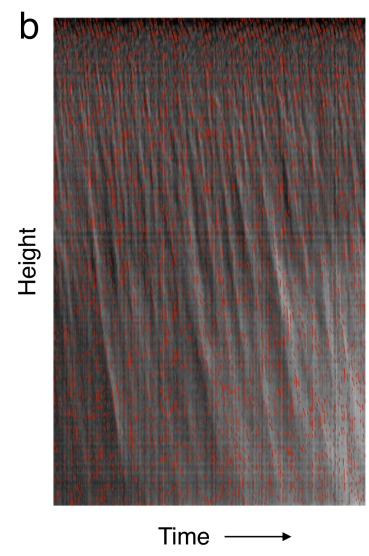

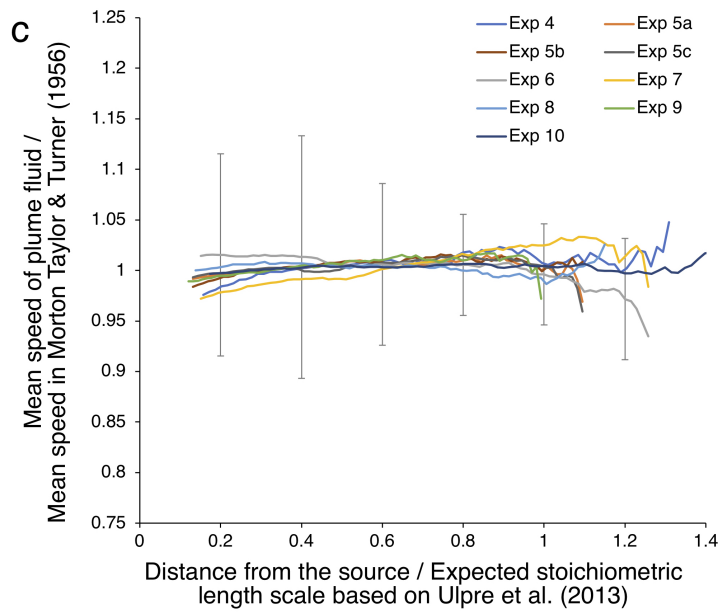

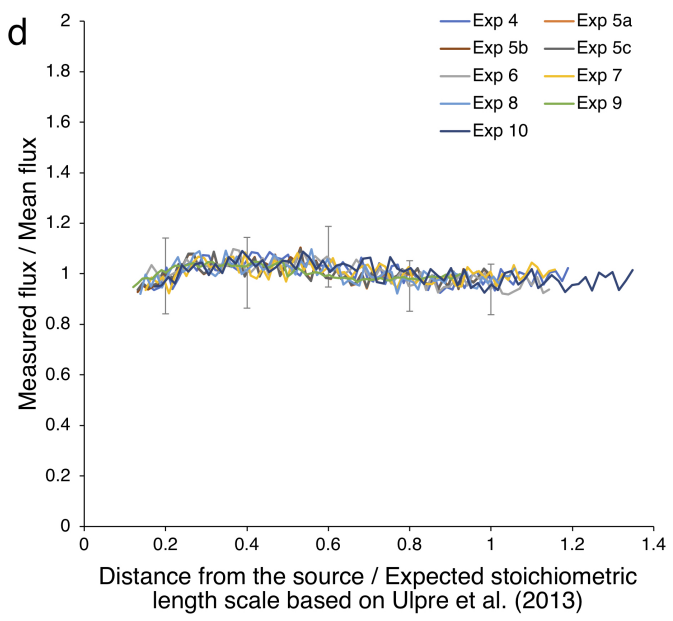

FiguRE 3. (a) For each image captured during an inert experiment, the horizontally-averaged profile of light attenuation is estimated across the width of the red box; (b) a time series of these profiles is plotted, and used to measure the characteristic speed of the descending eddies at different distances below the plume source; (c) the measured eddy velocity is compared with the top-hat speed $u=\left(6 \alpha \pi^{2} / 5 \lambda^{2}\right)^{-2}(B / z)^{1 / 3}$ (Morton et al. 1956), error bars are plotted for experiment 6 ; (d) the flux of light attenuation along the plume is estimated by multiplying the measured intensity of light attenuation produced by the plume fluid at a given distance from the source by the associated measured speed. It is seen that this flux is approximately constant, with deviations of order $5-10 \%$ associated with the limitations of the image analysis technique. This indicates that light attenuation across the tank is linearly proportional to the concentration of dye in the plume fluid.

respectively. Here, top-hat radial profiles are assumed (cf. Morton et al. 1956; Papanicolau \& List 1988), while $z_{0}(\mathrm{~m})$ denotes the vertical distance between the actual source and the virtual source of the plume (Hunt \& Kaye 2001) and $\alpha$ is the entrainment coefficient (Morton et al. 1956). To locate the virtual origin in our experiments, we first measured the time-averaged mean profiles of light attenuation along the plume's centreline in a number of experiments (figure 4a). Using equation 2.2 and the best fit of the results (figure $4 \mathrm{~b}$ ), we obtained $z_{0}=(5.3 \pm 0.2) \times 10^{-3} \mathrm{~m}$. For comparison, two filling box experiments were also performed in the tank using the method described by Linden et al. (1990), leading to 
a

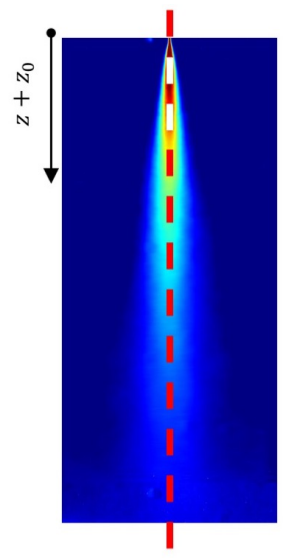

b

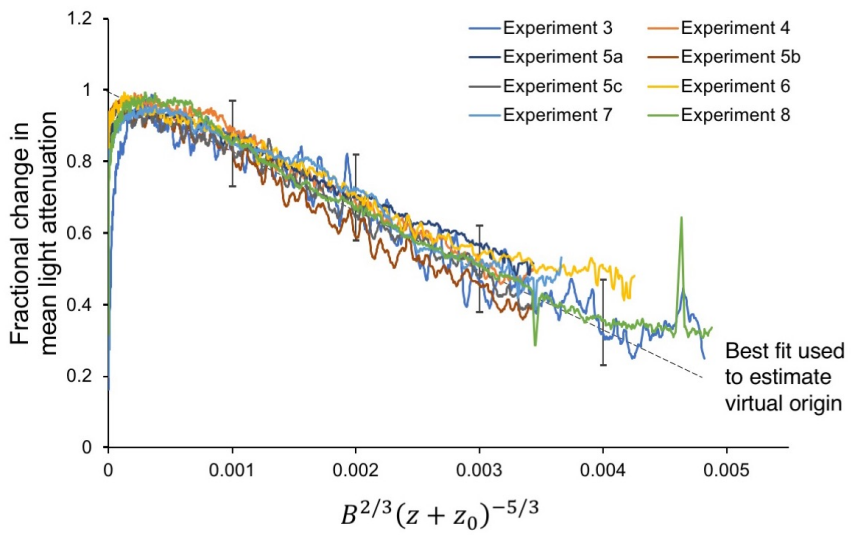

FiguRE 4. A number of time-averaged light attenuation profiles along the plume centreline are compared to estimate the virtual origin of the plume in our experimental setup.

a very similar result, $z_{0}=(5.2 \pm 0.3) \times 10^{-3} \mathrm{~m}$ (see Appendix A). For each experiment, the time-averaged image of the flow illustrated by figure 4a was used to measure the plume radius $b$ as a function of distance from the virtual source. At any particular height in the tank, we first measured the Gaussian width $b_{G}$ between the plume vertical axis and the points at which the time-averaged dye concentration is $1 / e$ of that on the axis (Morton et al. 1956). For conservation of mass and momentum, the top-hat radius of the plume was then defined as $b\left(z+z_{0}\right)=\sqrt{2} b_{G}\left(z+z_{0}\right)$. Equation 2.3 and our measurements of $b$ were used to estimate $\alpha=0.10 \pm 0.02$ in our experiments. Our estimates of both $z_{0}$ and $\alpha$ are in good agreement with the literature (e.g., Bower et al. 2008).

\section{Reactive systems}

\subsection{Fast reaction between acetic acid and ammonium hydroxide}

Two sets of experiments have been carried out to explore the behaviour of plumes with a fast chemical reaction. In the first set, an aqueous solution of acetic acid was used as the ambient fluid in the tank, and a solution of ammonium hydroxide was used as the plume fluid (see tables 1 and 2). The concentrations of chemicals used in this set of experiments are listed in table 2 and discussed in appendix B. Within the range of concentrations used, the chemical reaction between acetic acid and ammonium hydroxide had negligible effects on the density of the solutions (Domingos \& Cardoso 2015), and did not affect the entrainment coefficient $\alpha$ (see section 2.2 and appendix A). Inert sodium chloride was added to the plume fluid to increase its density relative to the ambient fluid. The products of the reaction between acetic acid and ammonium hydroxide are ammonium acetate and water

$$
\mathrm{CH}_{3} \mathrm{COOH}+\mathrm{NH}_{4} \mathrm{OH} \longrightarrow \mathrm{CH}_{3} \mathrm{COONH}_{4}+\mathrm{H}_{2} \mathrm{O}
$$

The rate law of this second order reaction is

$$
r=k_{r_{1}}\left[\mathrm{CH}_{3} \mathrm{COOH}\right]\left[\mathrm{NH}_{4} \mathrm{OH}\right]
$$

with $k_{r_{1}} \approx 10^{11} \mathrm{M}^{-1} \mathrm{~s}^{-1}$ at $20^{\circ} \mathrm{C}$ (Someya et al. 2009). Acetic acid and ammonium hydroxide are a weak acid and a weak base respectively, and therefore they are partly dissociated in their solutions, according to the following chemical equilibria (Housecroft 
\& Constable 2002)

$$
\mathrm{CH}_{3} \mathrm{COOH} \rightleftarrows \mathrm{CH}_{3} \mathrm{COO}^{-}+\mathrm{H}^{+}, \quad K_{\mathrm{CH}_{3} \mathrm{COOH}}=1.8 \times 10^{-5}
$$

and

$$
\mathrm{NH}_{3}+\mathrm{H}_{2} \mathrm{O} \rightleftarrows \mathrm{OH}^{-}+\mathrm{NH}_{4}{ }^{+}, \quad K_{\mathrm{NH}_{3}}=1.8 \times 10^{-5}
$$

It follows that the equilibrium constant for the reaction between acetic acid and ammonium hydroxide is given by

$$
\frac{K_{\mathrm{CH}_{3} \mathrm{COOH}} \times K_{\mathrm{NH}_{3}}}{K_{w}}=3.2 \times 10^{4}
$$

in which $K_{w}\left(\mathrm{M}^{2}\right)$ is the dissociation constant for ionised water,

$$
K_{w}=\left[\mathrm{OH}^{-}\right]\left[\mathrm{H}^{+}\right]=10^{-14}
$$

Since the constant given by equation 3.5 is large, the reaction is product-favoured and driven practically to completion (Housecroft \& Constable 2002).

To observe the motion of the plume fluid in the tank and track the progress of the reaction, a few drops of phenolphthalein indicator were added to the solution of ammonium hydroxide and sodium chloride just before the beginning of each experiment. Phenolphthalein is a weak acid, which reacts according to the following equilibrium (Housecroft \& Constable 2002)

$$
\text { HInd } \rightleftarrows \text { Ind }^{-}+\mathrm{H}^{+}
$$

In equation 3.7, HInd is the colourless acidic form of phenolphthalein, while Ind $^{-}$is the conjugate basic form, which is characterised by a pink/purple colour (Domingos \& Cardoso 2015). Hence, an alkaline solution containing phenolphthalein is coloured; however the colour fades when the $\mathrm{pH}$ of the solution decreases beyond neutralisation (Wittke 1983). The relationship between colour intensity and depletion of chemical is linear (Domingos \& Cardoso 2015).

\subsection{Fast reaction between nitric acid and sodium hydroxide}

In the second set of experiments involving a fast reaction between the plume and the ambient fluid, a solution of nitric acid was used as the ambient fluid in the tank, and a solution of sodium hydroxide was used as the plume fluid (see tables 1 and 3). Within the range of concentrations used, the chemical reaction between nitric acid and sodium hydroxide did not affect the density of the solutions (Ulpre et al. 2013), nor the entrainment coefficient $\alpha$ (see section 2.2 and appendix A). Just as in the first set of experiments, inert sodium chloride and a few drops of phenolphthalein indicator were added to the solution to control its density and colour. The products of the reaction are sodium nitrate and water (Ulpre et al. 2013)

$$
\mathrm{HNO}_{3}+\mathrm{NaOH} \longrightarrow \mathrm{NaNO}_{3}+\mathrm{H}_{2} \mathrm{O}
$$

Nitric acid and sodium hydroxide are a strong acid and a strong base respectively, and therefore we expect them to be completely dissociated in their solutions. The reaction given in equation 3.8 is instantaneous (Atkins 1978).

\subsection{Slower reaction between ascorbic acid and methylene blue}

A third set of experiments was carried out to observe the behaviour of plumes with a slower chemical reaction. In these experiments, a solution of methylene blue was used as the plume fluid, while a solution of ascorbic acid was used as the ambient fluid in 


$\begin{array}{ccccccccccc}\text { Exp. } & \begin{array}{c}Q_{0} \\ \left(\times 10^{-6}\right)\end{array} & \begin{array}{c}r_{0} \\ \left(\times 10^{-3}\right)\end{array} & \rho_{b}^{0} / \rho_{a} & \begin{array}{c}B \\ \left(\times 10^{-6}\right)\end{array} & \begin{array}{c}\Gamma_{0} \\ \left(\times 10^{-2}\right)\end{array} & \begin{array}{c}L_{M_{0}} \\ \left(\times 10^{-3}\right)\end{array} & \begin{array}{c}C_{b}^{0} \\ \left(\times 10^{-1}\right)\end{array} & D_{s} & L_{s} \\ 1 & 2.0 & 1.2 & 1.074 & 1.45 & 2.78 & 0.024 & 2.979 & 3.844 & 129.0 & 0.380 \\ 2 & 2.0 & 1.2 & 1.074 & 1.45 & 2.78 & 0.024 & 3.426 & 3.844 & 112.2 & 0.349 \\ 3 & 2.0 & 1.2 & 1.074 & 1.45 & 2.78 & 0.024 & 3.873 & 3.844 & 99.2 & 0.325 \\ 4 & 2.0 & 1.2 & 1.074 & 1.45 & 2.78 & 0.024 & 4.469 & 3.844 & 86.0 & 0.298 \\ 5 & 2.0 & 1.2 & 1.074 & 1.45 & 2.78 & 0.024 & 2.979 & 2.329 & 78.2 & 0.282 \\ 6 & 2.0 & 1.2 & 1.074 & 1.45 & 2.78 & 0.024 & 5.959 & 3.844 & 64.5 & 0.252 \\ 7 & 2.0 & 1.2 & 1.074 & 1.45 & 2.78 & 0.024 & 2.979 & 1.560 & 52.4 & 0.223 \\ 8 & 2.0 & 1.2 & 1.074 & 1.45 & 2.78 & 0.024 & 4.022 & 1.560 & 38.8 & 0.187 \\ 9 & 2.0 & 1.2 & 1.074 & 1.45 & 2.78 & 0.024 & 5.959 & 1.560 & 26.2 & 0.149 \\ 10 & 2.0 & 1.2 & 1.074 & 1.45 & 2.78 & 0.024 & 5.214 & 0.784 & 15.0 & 0.108 \\ 11 & 2.0 & 1.2 & 1.148 & 2.90 & 5.56 & 0.017 & 4.469 & 3.844 & 86.0 & 0.260 \\ 12 & 2.0 & 1.2 & 1.037 & 0.73 & 1.39 & 0.034 & 4.469 & 3.844 & 86.0 & 0.343\end{array}$

TABLE 2. Conditions for experiments (i): ammonium hydroxide and acetic acid (see section 3.1). We let $Q_{0}\left(\mathrm{~m}^{3} \mathrm{~s}^{-1}\right)$ denote the source volume flux, $r_{0}(\mathrm{~m})$ the radius of the nozzle, $\rho_{b}^{0} / \rho_{a}$ the ratio between the density of the plume fluid at the source and that of the ambient fluid, $B\left(\mathrm{~m}^{4}\right.$ $\mathrm{s}^{-3}$ ) the buoyancy flux. $\Gamma_{0}$ is the Morton number at the source (see Appendix A), while $L_{M_{0}}$ $(\mathrm{m})$ is the length scale for the near-source region in which the flow is affected by the supplied momentum flux (see Appendix A). $C_{a}(\mathrm{M})$ is the concentration of acetic acid in the ambient fluid, $C_{b}^{0}(\mathrm{M})$ is the concentration of ammonium hydroxide in the plume fluid at the source, $D_{s}$ is the stoichiometric dilution coefficient given by equation $5.12, L_{s}(\mathrm{~m})$ is the stoichiometric length scale given by equation 5.13. In our calculations, we use $\nu=1.0 \times 10^{-6}\left(\mathrm{~m}^{2} \mathrm{~s}^{-1}\right)$ as the kinematic viscosity of water at $20^{\circ} \mathrm{C}$ (Atkins 1978). In all experiments, the Reynolds number at the source is $R e_{0}=1061$, the Schmidt number associated with the plume fluid is $S c_{b}=502$ (Perry \& Green 2008), and that associated with the ambient fluid is $S c_{a}=829$ (Vitagliano \& Lyons 1956).

$\begin{array}{ccccccccccc}\text { Exp. } & \begin{array}{c}Q_{0} \\ \left(\times 10^{-6}\right)\end{array} & \begin{array}{c}r_{0} \\ \left(\times 10^{-3}\right)\end{array} & \rho_{b}^{0} / \rho_{a} & \begin{array}{c}B \\ \left(\times 10^{-6}\right)\end{array} & \begin{array}{c}\Gamma_{0} \\ \left(\times 10^{-2}\right)\end{array} & \begin{array}{c}L_{M_{0}} \\ C_{a} \\ \left(\times 10^{-3}\right)\end{array} & \begin{array}{c}C_{b}^{0} \\ \left(\times 10^{-1}\right)\end{array} & D_{s} & L_{s} \\ 13 & 2.0 & 1.2 & 1.074 & 1.45 & 2.78 & 0.024 & 0.343 & 0.384 & 112.2 & 0.349 \\ 14 & 2.0 & 1.2 & 1.074 & 1.45 & 2.78 & 0.024 & 0.298 & 0.233 & 78.2 & 0.282 \\ 15 & 2.0 & 1.2 & 1.074 & 1.45 & 2.78 & 0.024 & 0.298 & 0.156 & 52.4 & 0.223 \\ 16 & 2.0 & 1.2 & 1.074 & 1.45 & 2.78 & 0.024 & 0.596 & 0.156 & 26.2 & 0.149\end{array}$

TABLE 3. Conditions for experiments (ii): sodium hydroxide and nitric acid (see section 3.2). In all experiments, the Reynolds number at the source is $R e_{0}=1061$, the Schmidt number associated with the plume fluid is $S c_{b}=517$ (Noulty \& Leaist 1984), and that associated with the ambient fluid is $S c_{a}=337$ (Yeh \& Wills 1971).

$\begin{array}{ccccccccccc}\text { Exp. } & \begin{array}{c}Q_{0} \\ \left(\times 10^{-6}\right)\end{array} & \begin{array}{c}r_{0} \\ \left(\times 10^{-3}\right)\end{array} & \rho_{b}^{0} / \rho_{a} & \begin{array}{c}B \\ \left(\times 10^{-7}\right)\end{array} & \begin{array}{c}\Gamma_{0} \\ \left(\times 10^{-2}\right)\end{array} & L_{M_{0}} & C_{a} & \begin{array}{c}C_{b}^{0} \\ \left(\times 10^{-3}\right)\end{array} & D_{s} & L_{s} \\ 17 & 1.0 & 0.6 & 1.074 & 7.25 & 0.35 & 0.034 & 0.05 & 0.10 & 2.00 \times 10^{-3} & 0.016 \\ 18 & 1.0 & 0.6 & 1.074 & 7.25 & 0.35 & 0.034 & 0.10 & 0.10 & 9.99 \times 10^{-4} & 0.016 \\ 19 & 1.0 & 0.6 & 1.074 & 7.25 & 0.35 & 0.034 & 0.15 & 0.10 & 6.66 \times 10^{-4} & 0.016 \\ 20 & 1.0 & 0.6 & 1.074 & 7.25 & 0.35 & 0.034 & 0.20 & 0.10 & 5.00 \times 10^{-4} & 0.016 \\ 21 & 1.0 & 0.6 & 1.074 & 7.25 & 0.35 & 0.034 & 0.25 & 0.10 & 4.00 \times 10^{-4} & 0.016\end{array}$

TABle 4. Conditions for experiments (iii): methylene blue and ascorbic acid (see section 3.3). In all experiments, the Reynolds number at the source is $R e_{0}=1061$, the Schmidt number associated with the plume fluid is $S c_{b}=1209$ (Leaist 1988), and that associated with the ambient fluid is $S c_{a}=880$ (Shamim \& Baki 1980). 


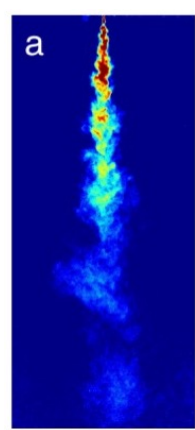

Inert

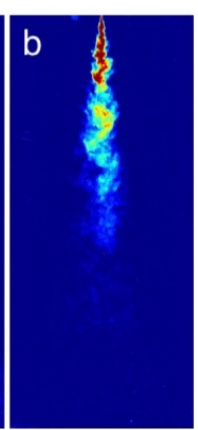

Reaction

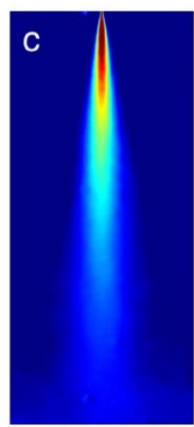

Inert

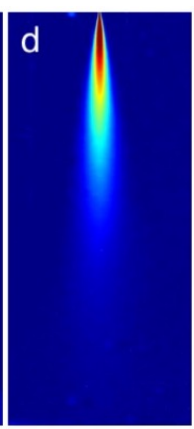

Reaction

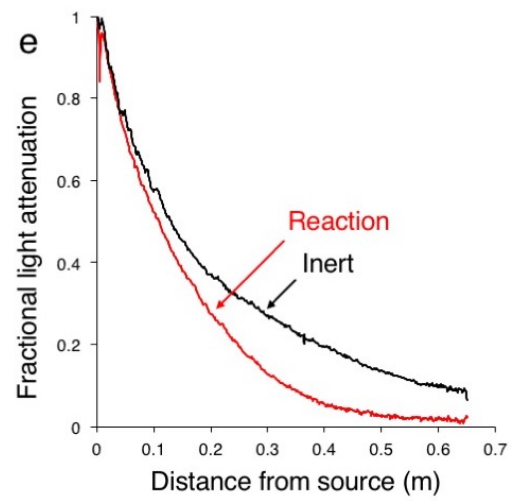

Figure 5. (a, b) Instantaneous and (c, d) time-averaged distributions of light attenuation produced by a plume of ammonium hydroxide descending through $(\mathrm{a}, \mathrm{c})$ fresh water or (b, d) a solution of acetic acid. A comparison of the time- and depth-averaged profiles of light attenuation along the plume's centreline is given in panel (e).

the tank (see tables 1 and 4). Within the range of concentrations used, the chemical reaction between ascorbic acid and methylene blue did not affect the density of the solutions (Domingos \& Cardoso 2015), nor the entrainment coefficient $\alpha$ (see section 2.2 and appendix A). Inert sucrose was added to the plume fluid to increase its density relative to the ambient fluid. The products of the reaction between methylene blue and ascorbic acid are leucomethylene blue and dehydroascorbic acid,

$$
\mathrm{MB}+\mathrm{H}_{2} \mathrm{~A} \longrightarrow \mathrm{LB}+\mathrm{DHA}
$$

Mowry \& Ogren (1999) measured the rate law of this second-order reaction

$$
r=k_{r_{2}}[\mathrm{MB}]\left[\mathrm{H}_{2} \mathrm{~A}\right]
$$

and found $k_{r_{2}} \approx 1 \pm 0.2 \mathrm{M}^{-1} \mathrm{~s}^{-1}$ at $20^{\circ} \mathrm{C}$. Snehalatha et al. (1997) proposed two different mechanisms for the reaction between ascorbic acid and methylene blue. Both mechanisms are irreversible and second order. As a result of the irreversible reaction, the colour of a mixture of methylene blue and ascorbic acid gradually fades. In our experiments, we track the progress of the reaction in the plume by observing the change in the colour of the plume fluid (see section 5.4).

It should be noted that for the reaction to produce significant effects within a relatively short time during each experiment, sufficiently large concentrations of ascorbic acid and methylene blue were used in this set of experiments (see table 4). At these concentrations, the colour of the plume fluid near the source was very dark, making it difficult to measure the concentration of dye in the plume eddies using the light attenuation technique in this region. However, the colour of the plume fluid rapidly became lighter as the fluid became increasingly dilute while descending through the tank. For this reason, in plotting figures 7d, 9 and 12 (sections 4 and 5), we will only consider the portion of the tank in which the concentration of dye in the plume fluid could be measured using the experimental technique described in section 2 .

\section{Experimental observations}

\subsection{Time-averaged concentration of the plume fluid}

Figure 5 shows a side-by-side comparison of the outcomes of two experiments in which the same alkaline plume fluid descended through either fresh water or an acidic solution 

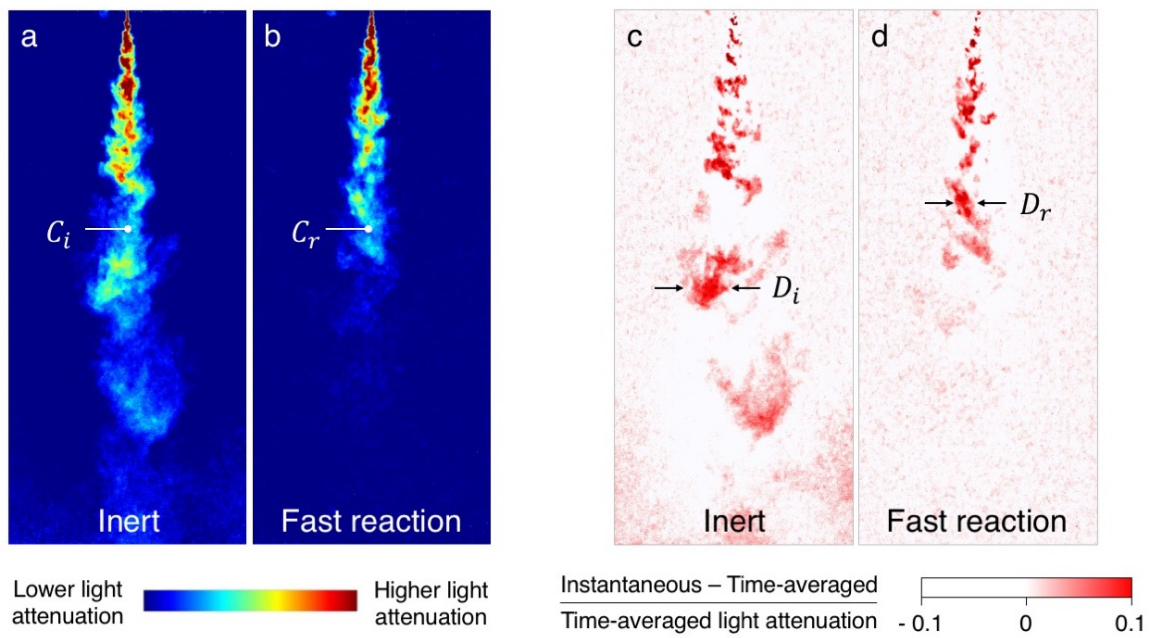

Figure 6. Instantaneous images of a fast-reaction, alkaline plume descending through (a) fresh water and (b) an acidic environment: a rainbow colormap is used to illustrate the distribution of the light attenuation in the tank. In figures (c) and (d), the same images are processed to highlight the descending eddies. A white/red colormap is used to highlight the regions of the plume in which the instantaneous dye concentration exceeds the time-averaged concentration.

(experiment 1 in table 2). In figures $5 \mathrm{a}$ and $\mathrm{c}$, the tank was filled with fresh water. Both the instantaneous and the time-averaged images show that while descending through the tank, the plume fluid became increasingly dilute as a result of the entrainment of ambient fluid. Figures 5b and d illustrate the results of an experiment in which the tank was filled with a solution of acetic acid. In this case, the plume was subjected to both dilution and chemical reaction, leading to a faster reduction in the $\mathrm{pH}$ of the fluid, which in turn resulted in a faster reduction in the concentration of dye in the fluid (figure 5e).

The red line in figure 5e shows that at distances below the source larger than approximately $0.45-0.50 \mathrm{~m}$, the plume in the reactive system produced a negligible amount of light attenuation through the tank. At this distance from the source, all the phenolphthalein dye contained in the supplied fluid had turned colourless, and this indicates that the $\mathrm{pH}$ of the fluid had decreased beyond neutralisation as a result of the chemical reaction (see section 2.1, cf. Wittke 1983).

\subsection{Distribution and concentration of the reactive fluid in plume eddies}

While comparing the time-averaged concentration profiles depicted in figure 5e enables us to identify the distance from the source at which the chemical reaction is complete, we should note that turbulent plumes are effectively composed of transient or intermittent eddies (Mingotti \& Woods 2015). It is interesting to observe how the concentration of the reactants varies in the eddies as a function of distance from the source and of the reaction rate.

In figure $6 \mathrm{a}$, we use false colours to illustrate the distribution of the light attenuation produced by a dyed inert plume which descends through fresh water. In order to identify the turbulent eddies in this picture, we first subtract the time-averaged mean distribution of light attenuation from the instantaneous distribution depicted in the image. We then use a red colourmap to illustrate the fractional amplitude of the fluctuations above the mean (figure 6c). We observe that as the inert plume fluid descends through fresh water, the diameter of the eddies, $D_{i}$, increases with distance from the source due to 

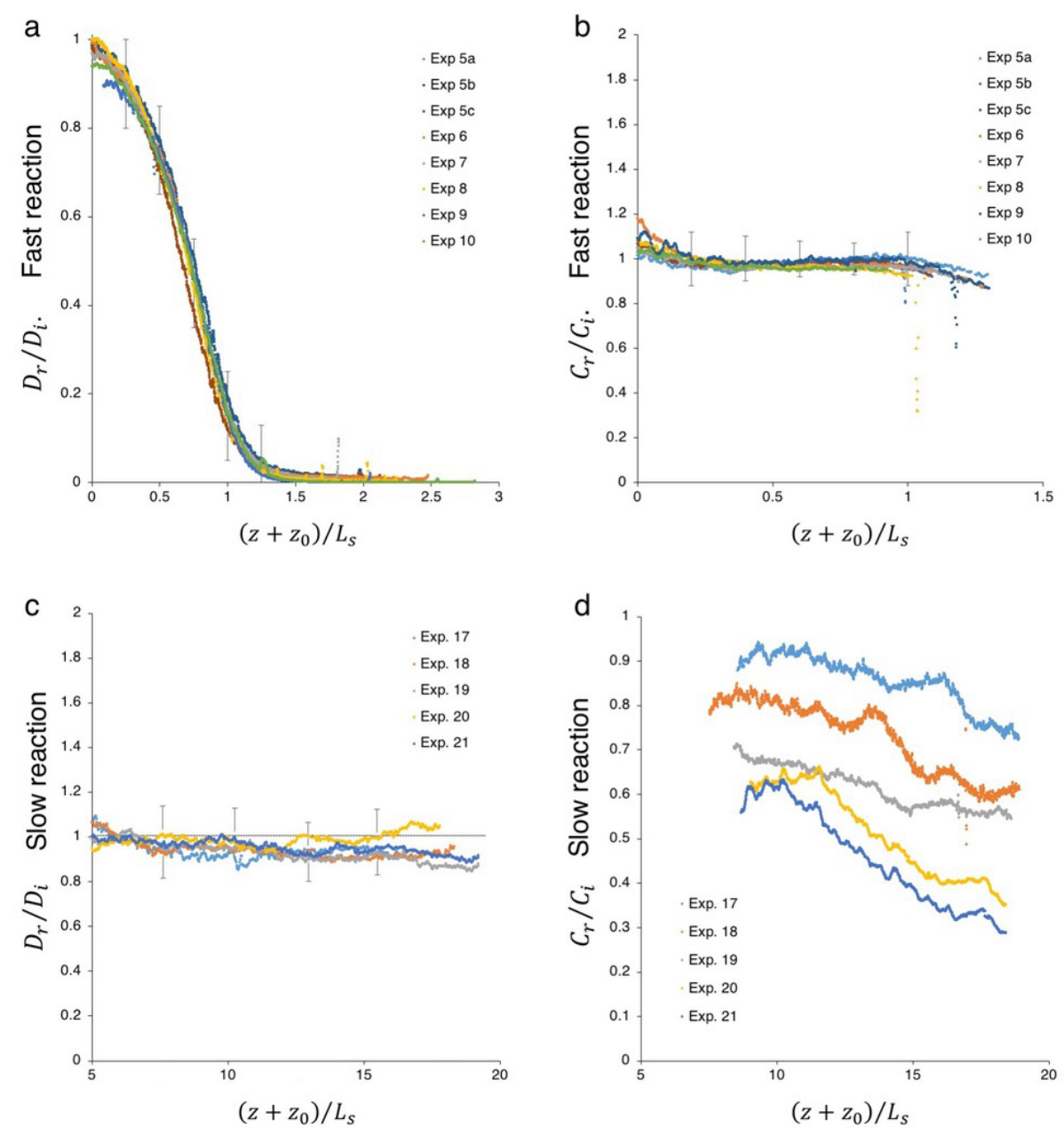

Figure 7. Time-averaged mean (a, c) size of the parcels of reactive fluid relative to the size of the eddies in an inert plume, $D_{r} / D_{i}$, and $(\mathrm{b}, \mathrm{d})$ concentration of dye per unit volume in a parcel of reactive fluid relative to the concentration in a parcel of inert plume fluid, $C_{r} / C_{i}$, for $(\mathrm{a}, \mathrm{b})$ fast and $(\mathrm{c}, \mathrm{d})$ slow reactions. All data are presented as a function of the distance from the virtual origin of the plume, scaled by the stoichiometric length scale, $L_{s}$, given by equation 5.13. Panels $(\mathrm{a}, \mathrm{b})$ show that the variation between the results of different experiments is larger at small distances below the source, $\left(z+z_{0}\right) / L_{s}<0.3-0.4$ (i.e. $z<4-6 \mathrm{~cm}$ below the nozzle approximately, see tables 2 and 3). In this near-source region, the flow in the plume is still transitional or just settling into full turbulence. It should be noted that the magnitude of the discrepancies between the results depicted in panels $(\mathrm{a}, \mathrm{b})$ is typically smaller than the expected errors associated with the image analysis technique, as discussed in section 2 .

the entrainment of ambient fluid (figure 6c, cf. Papanicolau \& List 1988), while the concentration of dye in the eddies, $C_{i}$, decreases as a result of dilution (figure $6 \mathrm{a}$ ).

We repeat the same analysis for all the images captured during each experiment: as an example, figures $6 \mathrm{~b}$ and $\mathrm{d}$ show the results obtained when processing one of the pictures captured during experiment 1 (fast reaction, see table 2). Using these figures, we measure the different sizes, $D_{r}$, and concentrations, $C_{r}$, of the parcels of dyed fluid in the reactive plume. Figure 7 illustrates the results of our measurements. It is seen that the mean diameter of the parcels of dyed fluid in a plume with a fast chemical reaction is similar to 
(a)

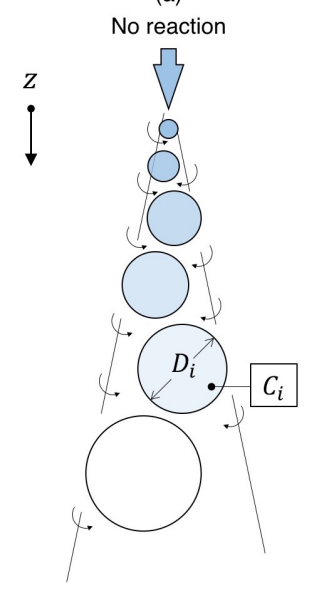

Reference plume (b)

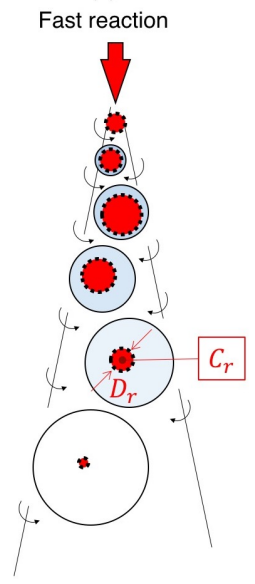

Reaction faster than mixing

$$
D_{r}<D_{i}
$$$$
C_{r} \approx C_{i}
$$

(c)

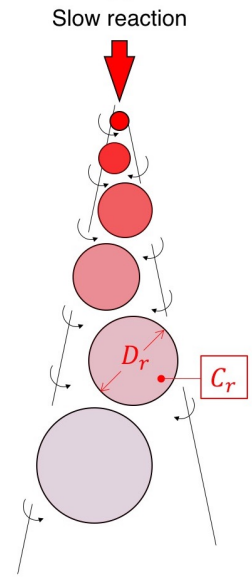

Mixing faster than reaction

$$
\begin{aligned}
& D_{r} \approx D_{i} \\
& C_{r}<C_{i}
\end{aligned}
$$

Figure 8. Cartoon illustrating the different distributions of reactants in the two cases of a fast and slow chemical reaction.

that of the eddies of an inert plume near the source; however, the ratio $D_{r} / D_{i}$ decreases progressively with distance from the source (figure 7a). On the other hand, we observe that the mean light attenuation produced by a unit volume of dyed fluid in a plume with a fast chemical reaction is approximately equal to that produced by a unit volume of dyed fluid in an inert plume at all distances from the source, $C_{r} / C_{i} \approx 1$ (figure $7 \mathrm{~b}$ ).

The results obtained when analysing the pictures captured during the slow reaction experiments are different. Figure 7c shows that the size of the parcels of dyed fluid in a plume with a slow reaction was similar to that of the eddies in an inert plume at all distances from the source in our experiments, $D_{r} / D_{i} \approx 1$. On the other hand, the concentration of dye in a plume with a slow reaction decreased progressively with distance from the source relative to the concentration of dye in an inert plume, $C_{r} / C_{i}<1$ (figure $7 \mathrm{~d})$.

These experimental observations suggest that the process of consumption of the chemical species in a plume with a fast reaction is different than that in a plume with a slower reaction (see figure 8). When the time required for the reaction to develop is smaller than the time required for a parcel of dyed plume fluid to mix with the entrained fluid, then the reaction primarily takes place near the edges of the parcel (dashed black lines in figure 8b). In this border region, the dyed fluid mixes locally with the entrained fluid: as the two fluids react, the colour of the dyed fluid rapidly fades. This leads to a reduction in the diameter of the parcel of dyed reactive fluid, $D_{r}$, as illustrated in figure 7a. The fluid in the core of the parcel, however, is not affected by the fast chemical reaction, and so its concentration $C_{r}$ is only subjected to the effects of dilution: hence, $C_{r}$ decreases with distance from the source at a rate similar to that of the concentration of dye in an inert plume, $C_{i}$ (see figure $7 \mathrm{~b}$ ).

On the other hand, in the case of a slower reaction, the time required for the convective mixing of the entrained fluid into an eddy is shorter than the time required for the reaction to develop. In this situation, the entrained reactants are continuously distributed 
throughout the entire volume of the eddy, $D_{r} \sim D_{i}$ (figures $7 \mathrm{c}$ and $8 \mathrm{c}$ ). The fluid in the bulk of the eddy is consequently subjected to the effects of both dilution and reaction, and so $C_{r}$ decreases more rapidly than $C_{i}$ with distance from the source (see figure $7 \mathrm{~d}$ ).

Motivated by these experimental results, in the next section we develop a simple model based on plume theory to quantify the concentration of the supplied reactants in the plume fluid as a function of distance from the source. We then test the model by comparing the outcome of the experiments with the model predictions.

\section{Model}

In this section, plume theory is used to build a model which quantifies the concentration of reactants in the plume fluid as a function of distance from the source in the two limiting cases of a slow and of an instantaneous reaction. In sections 5.1-5.3, we first estimate and compare the length and time scales which govern the processes of entrainment, dilution and chemical reaction within the plume. In section 5.4, we focus on relatively slow reactions, which develop on a time scale larger than that required for the convective mixing of the fluid in the plume. In section 5.5, we focus on fast reactions. We estimate the fraction of the entrained volume flux which mixes and reacts within the plume as a function of distance from the source. In the limit of an instantaneous reaction, we calculate the distance at which the reaction is complete.

\subsection{Entrainment of ambient fluid}

Plume theory indicates that as a result of entrainment of ambient fluid, the plume volume flux, $Q\left(\mathrm{~m}^{3} \mathrm{~s}^{-1}\right)$, increases with distance from the source $z$ (Morton et al. 1956) as

$$
Q=\lambda B^{\frac{1}{3}} z^{\frac{5}{3}}
$$

where $B\left(\mathrm{~m}^{4} \mathrm{~s}^{-3}\right)$ is the buoyancy flux and

$$
\lambda=\frac{6}{5} \alpha\left(\frac{9}{10} \alpha\right)^{\frac{1}{3}} \pi^{\frac{2}{3}}
$$

is a universal constant dependent on the entrainment coefficient $\alpha$ (Linden 1990). Both the plume radius $b$ and the mean diameter of the eddies in the plume increase linearly with distance from the source (Papanicolau \& List 1988). Consequently, the mean speed of the fluid, $u\left(\mathrm{~m} \mathrm{~s}^{-1}\right)$, decreases with distance from the source

$$
u=\frac{Q}{\pi b^{2}} \sim \alpha^{-2} \lambda B^{\frac{1}{3}} z^{-\frac{1}{3}}
$$

It follows that the turbulent motion of the plume fluid and the entrainment of ambient fluid into the plume are governed by the following length and time scales (cf. table 5)

$$
L_{e}=\alpha z
$$

and

$$
\tau_{e}=\frac{L_{e}}{u}=\alpha^{3} \lambda^{-1} B^{-\frac{1}{3}} z^{\frac{4}{3}}
$$

\subsection{Stoichiometric dilution of the supplied fluid}

We let $Q_{0}\left(\mathrm{~m}^{3} \mathrm{~s}^{-1}\right)$ denote the plume volume flux at the source. We assume that the supplied fluid contains an alkaline solution, $\mathrm{MOH}$, and we let $C_{b}^{0}(\mathrm{M})$ denote the initial concentration of this solution. As the plume descends through the surrounding ambient, 


$\begin{array}{lll} & \text { Length scale } L & \text { Time scale } \tau \\ \text { Entrainment } & \alpha z & \alpha^{3} \lambda^{-1} B^{-\frac{1}{3}} z^{\frac{4}{3}} \\ \text { Mixing } & S c_{b}^{-\frac{1}{2}} \alpha^{\frac{7}{4}} \lambda^{-\frac{3}{4}} \nu^{\frac{3}{4}} B^{-\frac{1}{4}} z^{\frac{1}{2}} & \ln S c_{b} \cdot \alpha^{\frac{7}{2}} \lambda^{-\frac{3}{2}} \nu^{\frac{1}{2}} B^{-\frac{1}{2}} z \\ \text { Stoichiometry } & \lambda^{-\frac{3}{5}} Q_{0}^{\frac{3}{5}} B^{-\frac{1}{5}}\left(1+D_{s}\right)^{\frac{3}{5}} & \alpha^{2} \lambda^{-\frac{8}{5}} Q_{0}^{\frac{3}{5}} B^{-\frac{8}{15}} z^{\frac{1}{3}}\left(1+D_{s}\right)^{\frac{3}{5}} \\ \text { Reaction } & \alpha^{-2} \lambda B^{\frac{1}{3}} z^{-\frac{1}{3}}\left(k_{r} C_{a}\right)^{-1} & \left(k_{r} C_{a}\right)^{-1}\end{array}$

TABLE 5. Length and time scales used in the model.

the supplied fluid is diluted through the addition of a volume flux $Q_{e}$ of entrained acid, HA, of a uniform concentration $C_{a}$. As indicated by Ulpre et al. (2013), the chemical reaction between the fluids is governed by the conservation of charge

$$
\left[\mathrm{H}^{+}\right]+\left[\mathrm{M}^{+}\right]=\left[\mathrm{OH}^{-}\right]+\left[\mathrm{A}^{-}\right]
$$

and by the conservation of mass of acid and alkali respectively

$$
C_{a} Q_{e}=\left([\mathrm{AH}]+\left[\mathrm{A}^{-}\right]\right)\left(Q_{e}+Q_{0}\right)
$$

$$
C_{b}^{0} Q_{0}=\left([\mathrm{MOH}]+\left[\mathrm{M}^{+}\right]\right)\left(Q_{e}+Q_{0}\right)
$$

We let $K_{a}$ and $K_{b}(\mathrm{M})$ denote the dissociation constants of the acidic and of the alkaline solutions respectively

$$
K_{a}=\frac{\left[\mathrm{A}^{-}\right]\left[\mathrm{H}^{+}\right]}{[\mathrm{AH}]}
$$

$$
K_{b}=\frac{\left[\mathrm{M}^{+}\right]\left[\mathrm{OH}^{-}\right]}{[\mathrm{MOH}]}
$$

We define the dilution coefficient $D$ as the ratio between the volume flux of the entrained fluid and that of the supplied fluid at a given distance from the source. Using a combination of equations 5.7-5.10, this coefficient is given by

$$
D=\frac{C_{b}^{0} K_{b}\left[\mathrm{~A}^{-}\right]\left(K_{a}+\left[\mathrm{H}^{+}\right]\right)}{C_{a} K_{a}\left[\mathrm{M}^{+}\right]\left(K_{b}+\left[\mathrm{OH}^{-}\right]\right)}
$$

As the volume flux of entrained fluid increases with distance from the source, the number of moles of entrained acid and the dilution coefficient also increase. We let $D_{s}$ denote the critical stoichiometric dilution at which the supplied alkali and entrained acid are present in the plume in stoichiometric amounts. At this critical dilution, the mean concentration of hydrogen ions equals that of hydroxide ions in the solution, $\left[\mathrm{H}^{+}\right]=\left[\mathrm{OH}^{-}\right]$, and so using equations 3.6 and 5.6 we obtain

$$
D_{s}=\frac{C_{b}^{0} K_{b}\left(K_{a}+K_{w}^{1 / 2}\right)}{C_{a} K_{a}\left(K_{b}+K_{w}^{1 / 2}\right)}
$$

Using a combination of equations 5.1 and 5.12 , we calculate that the distance between the plume source and the level at which the stoichiometric dilution is achieved is given 
by

$$
L_{s}=\lambda^{-\frac{3}{5}} Q_{0}^{\frac{3}{5}} B^{-\frac{1}{5}}\left(1+D_{s}\right)^{\frac{3}{5}}
$$

as listed in table 5. Equations 5.3 and 5.13 are then used to estimate that the time required for the plume fluid to flow from the source to the stoichiometric level scales by

$$
\tau_{s}=\frac{L_{s}}{u}=\alpha^{2} \lambda^{-\frac{8}{5}} Q_{0}^{\frac{3}{5}} B^{-\frac{8}{15}} z^{\frac{1}{3}}\left(1+D_{s}\right)^{\frac{3}{5}}
$$

\subsection{Time scale for chemical reaction}

In our experiments, the volumetric flow rate of ambient fluid entrained into the plume becomes larger than that of source fluid at short distances from the source. The concentration of the ambient chemical species in the plume is therefore approximately constant in the plume, and the chemical reaction between the source and the entrained fluid may be treated as a pseudo-first order reaction (Domingos \& Cardoso 2015). The time scale for a change in the concentration of the chemical species in the plume fluid is therefore given by (see table 5)

$$
\tau_{r}=\left(k_{r} C_{a}\right)^{-1}
$$

while the distance travelled by the plume fluid during this time scales as

$$
L_{r}=u \tau_{r}=\alpha^{-2} \lambda B^{\frac{1}{3}} z^{-\frac{1}{3}}\left(k_{r} C_{a}\right)^{-1}
$$

We compare the time scale for achieving the stoichiometric dilution, $\tau_{s}$, with the reaction time scale, $\tau_{r}$, in our experiments. In the slow reaction experiments (see section 3.3 and table 4 ), the ratio $\tau_{s} / \tau_{r}$ is typically of order $10^{-3}-10^{-2}$. This indicates that the plume rapidly entrains a large amount of ambient fluid while descending to the stoichiometric level $L_{s}$; however, the chemical reaction is comparatively slow. Hence, we expect the consumption of the supplied chemicals to be controlled by the reaction time scale (see table 5), and most of the effects of the reaction to be visible at distances larger than $L_{s}$. On the other hand, in our fast reaction experiments (see sections 3.13.2 and tables $2-3), \tau_{s} / \tau_{r}$ is typically of order $10^{7}-10^{8}$. This suggests that as soon as a parcel of acidic fluid is entrained from the ambient and mixed within the plume, it immediately reacts with the descending fluid. Hence, we expect the consumption of the supplied chemicals to be controlled by the entrainment and mixing time scales (see table 5 ), and most of the effects of the reaction to be visible at distances smaller than $L_{s}$.

\subsection{Consumption of the chemical species in a plume with a slow reaction}

In this section, we focus on slow reactions and quantify the concentration of the supplied chemicals in the plume fluid as a function of distance from the source. Both dilution and reaction lead to a gradual reduction in the concentration of the supplied chemical species $C_{b}$ (cf. Domingos \& Cardoso 2013). Using equations 5.1 and 5.15, this concentration varies with time according to

$$
\frac{d C_{b}}{d t}=-\frac{5}{4} \frac{C_{b}}{t}-\frac{C_{b}}{\tau_{r}}
$$

The ratio between the concentration in a reactive system $\left(C_{a}>0, \tau_{r}>0\right)$ and that in an inert system $\left(C_{a}=0, \tau_{r} \rightarrow \infty\right)$ is then

$$
\frac{C_{b, \text { reaction }}}{C_{b, \text { inert }}}=\exp \left(-\frac{t}{\tau_{r}}\right)
$$

Our model of the consumption of reactants in a plume with a slow chemical reaction is 


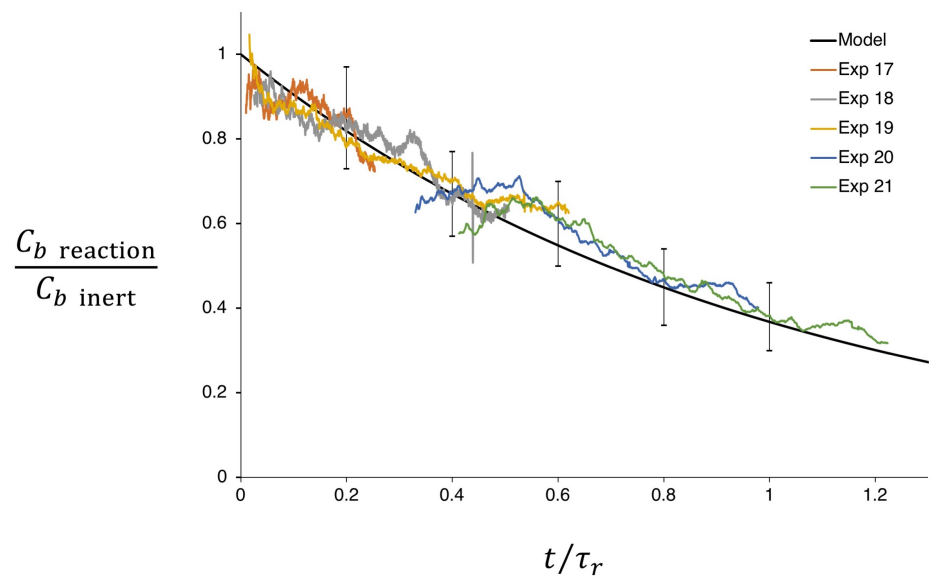

FIGURE 9. Comparison between the results of the slow reaction experiments (coloured lines) and the prediction of the model (black line, based on equation 5.18).

tested against the results of the laboratory experiments described in section 3.3. For each of the experiments listed in table 4 , we calculate the ratio between the time-averaged light attenuation produced by a methylene blue plume which descends through an acidic environment, and that produced by the associated inert plume which descends through fresh water (cf. figure 7d). Figure 9 shows that the model prediction (black line, based on equation 5.18) is in good agreement with the results of the experiments, with deviations of order $10 \%$, which are compatible with the expected errors associated with the image processing technique (see section 2.2).

\subsection{Consumption of the chemical species in a plume with a fast reaction}

We now consider the case of a fast reaction between the supplied and the entrained fluid in the plume. In the limit $\tau_{s} / \tau_{r} \gg 1$, we assume the reaction to be instantaneous $\left(\tau_{r}=L_{r}=0\right)$, and the supplied chemical species to be progressively consumed in the region $0<z<L_{s}$ as an increasing flux of ambient fluid is entrained and mixed within the plume.

Domingos \& Cardoso (2015) previously observed non-uniform distributions of reactants in single-phase thermals with a fast reaction, and found the progress of the reaction to be controlled by the local mixing of the reactive fluids. Similarly, in section 4.2 we observed that sufficiently fast reactions may only develop in a portion of the plume's volume, with chemically active regions near the edges of each parcel of reactive fluid, and inactive regions in the core of each parcel of fluid (see figure 8b). Based on these experimental observations and on the previous findings by Domingos \& Cardoso, we let $\tau_{m}$ denote the time scale for the local mixing of the fluids at the Batchelor scale (Batchelor 1959; Fox 2003),

$$
\tau_{m}=\frac{1}{2} \ln S c_{b} \tau_{e} R e^{-\frac{1}{2}} \sim \ln S c_{b} \cdot \alpha^{\frac{7}{2}} \lambda^{-\frac{3}{2}} \nu^{\frac{1}{2}} B^{-\frac{1}{2}} z
$$

in which $S c_{b}$ is the Schmidt number associated with the plume (see tables 2 and 3), $\nu$ $\left(\mathrm{m}^{2} \mathrm{~s}^{-1}\right)$ is the kinematic viscosity of the mixture (Pope 2000; Kundu et al. 2015), and

$$
R e=\frac{u L_{e}}{\nu}=\alpha^{-1} \lambda \nu^{-1} B^{\frac{1}{3}} z^{\frac{2}{3}}
$$

is the Reynolds number associated with the plume eddies. For each of our experiments, we calculate $\tau_{m}$ as a function of distance from the source using equation 5.19 and the 
18

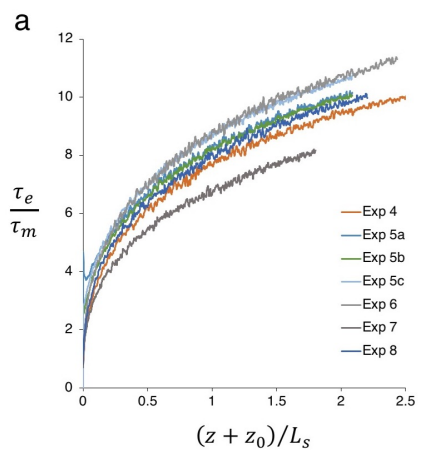

N. Mingotti and S. S. S. Cardoso

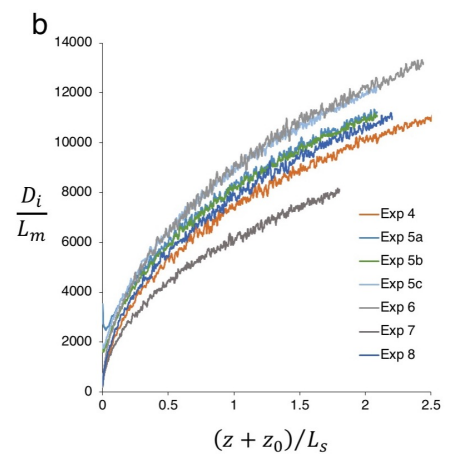

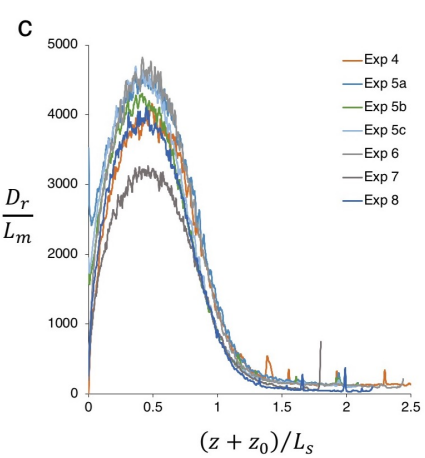

Figure 10. Experimental measurements of (a) the ratio between the time scale for convective mixing of the fluid in an eddy and the Batchelor time scale, $\tau_{e} / \tau_{m}$ (Batchelor 1959; Fox 2003); (b) the ratio between the time-averaged mean diameter of the eddies and the Batchelor length scale, $D_{i} / L_{m}$; and (c) the ratio between the time-averaged mean diameter of the parcels of reactive fluid and the Batchelor length scale, $D_{r} / L_{m}$. All data are presented as a function of the distance from the virtual origin of the plume, scaled by $L_{s}$ (equation 5.13).

experimental measurements of the speed and size of the eddies in the plume (see section 2.2). Figure 10a shows that the time required for the turbulent convection of the fluid in an eddy is always much larger than the time required for the local mixing of the fluid at the Batchelor scale in our experiments, $\tau_{e} \gg \tau_{m}$. Hence, we expect that fast chemical reactions will develop primarily at this latter scale, and that the depth of the chemically active region in the periphery of each parcel of reactive fluid will be controlled by the Batchelor length scale, $L_{m}$ (Batchelor 1959; Fox 2003)

$$
L_{m}=S c_{b}^{-\frac{1}{2}} L_{e} R e^{-\frac{3}{4}}=S c_{b}^{-\frac{1}{2}} \alpha^{\frac{7}{4}} \lambda^{-\frac{3}{4}} \nu^{\frac{3}{4}} B^{-\frac{1}{4}} z^{\frac{1}{2}}
$$

For each experiment with a fast reaction, $L_{m}$ is estimated using equation 5.21 and the experimental measurements of the time-averaged speed and size of the eddies in the plume (see section 2.2). In figures 10b and c, we compare $L_{m}$ with the mean diameters of the eddies, $D_{i}$, and of the parcels of reactive fluid in our experiments, $D_{r}$ (see figure 6). It is seen that in the region $0<z<L_{s}$, the Batchelor length scale is much smaller than both diameters: this is consistent with our experimental observation of the chemical reaction developing in a relatively thin region near the edge of each parcel of reactive fluid in the plume (see figure 8b). The mixture of ambient and entrained fluid in this region rapidly becomes neutralised, leading to a reduction in the parcel's size (see figure 7). As a result of the slower process of large-scale mixing, some of the neutralised fluid is subsequently entrained and diluted into the core of the parcel of fluid; however, we expect that no chemical reaction will develop there.

Using equations 5.19 and 5.21, we estimate that the volume flux of entrained fluid which mixes with the plume fluid in a unit of time, herein denoted by $Q_{m}$, scales by

$$
\frac{d Q_{m}}{d t} \sim \frac{L_{m}^{3}}{\tau_{m}^{2}}=\frac{\alpha^{-\frac{7}{4}} \lambda^{\frac{3}{4}} \nu^{\frac{5}{4}} B^{\frac{1}{4}} z^{-\frac{1}{2}}}{S c_{b}^{\frac{3}{2}}\left(\ln S c_{b}\right)^{2}}
$$

In turn, plume theory indicates that the volume flux of ambient fluid which is entrained by the plume in a unit of time increases with distance from the source according to

$$
\frac{d Q}{d t} \sim \frac{L_{e}^{3}}{\tau_{e}^{2}}=\alpha^{-3} \lambda^{2} B^{\frac{2}{3}} z^{\frac{1}{3}}
$$

At all times, the volume flux of entrained fluid which mixes within the plume must be 
smaller or equal to the volume flux of fluid which is entrained from the ambient,

$$
\frac{d Q_{m}}{d t} \leqslant \frac{d Q}{d t}
$$

Using equations 5.22 and 5.23 , we estimate that the fraction of the entrained volume flux which mixes and reacts with some of the supplied fluid in the plume decreases with distance from the source

$$
\frac{d Q_{m}}{d Q}=\min \left(\frac{\alpha^{\frac{5}{4}} \beta \lambda^{-\frac{5}{4}} \nu^{\frac{5}{4}} B^{-\frac{5}{12}} z^{-\frac{5}{6}}}{S c_{b}^{\frac{3}{2}}\left(\ln S c_{b}\right)^{2}} ; 1\right)
$$

in which $\beta$ is a dimensionless constant. Equation 5.25 indicates that a critical distance from the source exists, at which the volume flux of fluid which is entrained by the plume is equal to that which is mixed by the small-scale turbulence

$$
z_{\text {crit }}=\left(\frac{\beta^{3}}{S c_{b}^{2}\left(\ln S c_{b}\right)^{6}}\right)^{\frac{2}{5}} \frac{L_{m}^{2}}{L_{e}}
$$

For $z<z_{\text {crit }}$, the volume of fluid entrained by the plume is smaller than the maximum volume which can be mixed at the Batchelor scale: we therefore expect that all the fluid entrained by the plume will mix with a portion of the supplied plume fluid and react, but the overall progress of the reaction will be limited by the lack of entrained fluid. On the other hand, for $z>z_{\text {crit }}$ the volume of fluid which is entrained by the plume is larger than the maximum volume which can be mixed at the Batchelor scale: in this case, the reaction will only develop in a fraction of the entrained fluid due to incomplete mixing.

Equation 5.26 suggests that in case $z_{\text {crit }}<L_{s}$, only a fraction of the entrained fluid will react with the supplied fluid in the region $z_{\text {crit }}<z<L_{s}$. Consequently, the consumption of the chemical species will be reduced in this region, and the distance from the source at which the chemical reaction reaches completion, $z_{r}$, will be larger than $L_{s}$ (equation 5.13). To estimate this distance, we let $R$ denote the fraction of the supplied chemicals which are still reactive at a given distance from the source. Using equations 5.12, 5.25 and 5.26 , we calculate that this fraction is given by

$$
R(z)= \begin{cases}1-\frac{Q(z)}{\left(1+D_{s}\right) Q_{0}}, & 0<z<z_{\text {crit }} \\ 1-\frac{1}{\left(1+D_{s}\right) Q_{0}}\left(\lambda B^{\frac{1}{3}} z_{\text {crit }}^{\frac{5}{3}}+\int_{z_{\text {crit }}}^{z} \frac{\alpha^{\frac{5}{4}} \beta \nu^{\frac{5}{4}} B^{-\frac{5}{12}} z^{-\frac{5}{6}}}{\lambda^{\frac{5}{4}} S c_{b}^{\frac{3}{2}}\left(\ln S c_{b}\right)^{2}} \frac{d Q}{d z} d z\right), & z_{\text {crit }}<z<z_{r} .\end{cases}
$$

A combination of equations 5.1, 5.13 and 5.27 enables us to write $R$ as a function of the stoichiometric length scale, $L_{s}$, and the critical distance, $z_{\text {crit }}$

$$
R(z)= \begin{cases}1-\left(\frac{z}{L_{s}}\right)^{\frac{5}{3}}, & 0<z<z_{\text {crit }} \\ 1+\left(\frac{z_{\text {crit }}}{L_{s}}\right)^{\frac{5}{3}}-2\left(\frac{z}{L_{s}}\right)^{\frac{5}{6}}\left(\frac{z_{\text {crit }}}{L_{s}}\right)^{\frac{5}{6}}, & z_{\text {crit }}<z<z_{r} .\end{cases}
$$

Equation 5.28 indicates that the fraction of reactive chemicals in the plume decreases more rapidly in the region $0<z<z_{\text {crit }}$, where all of the entrained fluid mixes with the descending fluid and reacts; however, for $z_{\text {crit }}<z<z_{r}$, some of the entrained fluid does not react due to incomplete mixing, and so $R$ decreases more slowly in this region. Using equation 5.27, we calculate that the distance from the source at which the instantaneous 
reaction reaches completion $(R=0)$ is given by

$$
z_{r}=\left[\frac{\left(1+D_{s}\right) Q_{0}+\alpha^{\frac{5}{2}} \beta^{2} \lambda^{-\frac{3}{2}} S c_{b}^{-3}\left(\ln S c_{b}\right)^{-4} \nu^{\frac{5}{2}} B^{-\frac{1}{2}}}{2 \alpha^{\frac{5}{4}} \beta \lambda^{-\frac{1}{4}} S c_{b}^{-\frac{3}{2}}\left(\ln S c_{b}\right)^{-2} \nu^{\frac{5}{4}} B^{-\frac{1}{12}}}\right]^{\frac{6}{5}}
$$

The ratio between this distance and the stoichiometric length scale is then obtained from equation 5.28

$$
\frac{z_{r}}{L_{s}}=2^{-\frac{6}{5}}\left[\left(\frac{z_{\text {crit }}}{L_{s}}\right)^{\frac{5}{6}}+\left(\frac{z_{\text {crit }}}{L_{s}}\right)^{-\frac{5}{6}}\right]^{\frac{6}{5}}
$$

As expected, equation 5.30 indicates that $z_{r} / L_{s}=1$ when $z_{\text {crit }}=L_{s}$ so that all the fluid entrained by the plume mixes with the supplied fluid while descending from the source to the stoichiometric level. However, for $z_{\text {crit }}<L_{s}$ some of the entrained fluid does not immediately react within the plume, and this results in an increase in the distance required for the consumption of the supplied chemical species, $z_{r} / L_{s}>1$.

Our model of the consumption of reactants in a plume with a fast chemical reaction is tested against the results of the laboratory experiments described in sections 3.1 and 3.2. In doing so, we first use our measurements of the mean size of the inert and reactive parcels of fluid in the plume $\left(D_{i}\right.$ and $D_{r}$ respectively, see figure $\left.7 \mathrm{a}\right)$ to estimate the value of the dimensionless coefficient $\beta$ introduced in equation 5.25. We then use this value and equation 5.30 to calculate the expected level at which the chemical reaction reaches completion, and we compare the prediction of the model with the outcome of the experiments.

While estimating the value of $\beta$ in our experiments, we assume as an approximation that the volume of each parcel of fluid in the plume is proportional to the third power of the diameter of the parcel. It follows that as the plume entrains fluid from the surrounding ambient, the mean diameter of the plume eddies, $D_{i}$, increases with distance from the source according to

$$
\frac{d Q}{d z} \approx \frac{1}{\tau_{e}} \frac{d D_{i}^{3}}{d z}
$$

A fraction of the entrained fluid mixes and reacts within the plume, leading to a reduction in the mean diameter of the parcels of reactive fluid (see figure 8b). Using equations 5.12 and 5.22, we estimate that $D_{r}$ decreases with distance from the source according to

$$
\left(\frac{1+D_{s}}{D_{s}}\right) \frac{d Q_{m}}{d z} \approx-\frac{4 \pi D_{r}^{2}}{\tau_{m}} \frac{d D_{r}}{d z}
$$

Using a combination of equations 5.25, 5.31 and 5.32, we obtain

$$
\frac{\beta}{S c_{b}^{\frac{3}{2}}\left(\ln S c_{b}\right)^{2}}\left(\frac{\alpha^{\frac{5}{4}} \nu^{\frac{5}{4}}}{\lambda^{\frac{5}{4}} B^{\frac{5}{12}} z^{\frac{5}{6}}}\right) \approx-\frac{4}{3} \frac{\pi D_{s}}{\left(1+D_{s}\right)}\left(\frac{D_{r}^{2} \tau_{e}}{D_{i}^{2} \tau_{m}}\right) \frac{d D_{r}}{d D_{i}}+c_{1}
$$

Equation 5.33 and the results of the experiments described in sections 3.1 and 3.2 are used to plot figure 11a. It is seen that for $z_{\text {crit }}<z<z_{r}$, the data collected during a number of different experiments collapse onto a line: by measuring the slope of this line, we estimate that $\beta S c_{b}^{-\frac{3}{2}}\left(\ln S c_{b}\right)^{-2} \approx(2.0 \pm 0.14) \times 10^{4}$ in our experiments. It should be noted that since equations 5.31-5.33 are based on the approximation that the volume of each parcel of fluid in the plume is proportional to the third power of the diameter of the parcel and involve a derivative of the experimental measurements, we should not expect the collapse of the experimental results depicted in figure 11a to be perfect. Furthermore, 
a

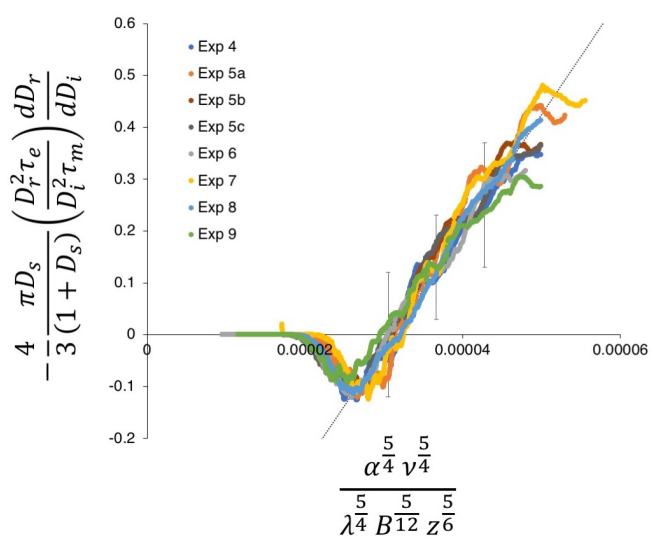

b

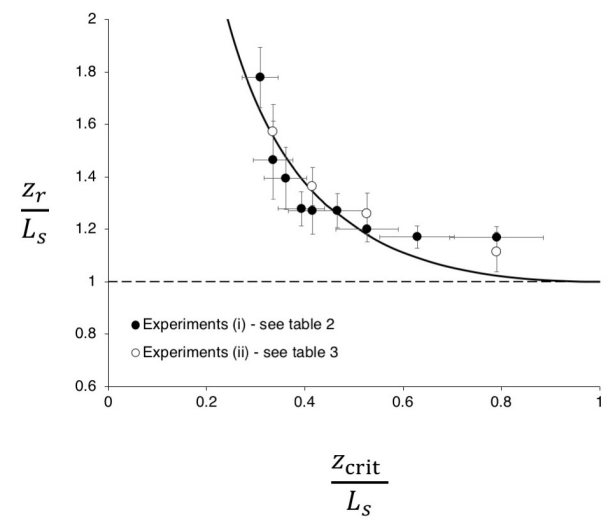

Figure 11. (a) We use equation 5.33 to estimate the value of $\beta$ in our experiments, based on the measurements of $D_{i}$ and $D_{r}$ illustrated in figure 7; (b) Equation 5.30 and the measured constant $\beta S c_{b}^{-3 / 2}\left(\ln S c_{b}\right)^{-2}$ are then used to estimate the distance below the source at which the instantaneous acid-base reaction is complete, $z_{r}$ (solid line). Close and open circles are used to illustrate the results of experiments (i) and (ii) respectively (see tables 3 and 2). In plotting the symbols, horizontal error bars are included to illustrate the impacts of a $\pm 10 \%$ deviation in the value of $\beta$.

equation 5.33 only applies to the region of the flow in which the mixing of the entrained fluid is incomplete, and is not applicable to the near-source region, $0<z<z_{\text {crit }}$ (see equation 5.25). For this reason, we do not expect the vertical intercepts of the sloping lines in figure 11a to be equal to zero, and a dimensionless constant $c_{1}$ has been added to equation 5.33 accordingly. Since both the buoyancy flux $B\left(\mathrm{~m}^{4} \mathrm{~s}^{-3}\right)$ and the viscosity $\nu$ $\left(\mathrm{m}^{2} \mathrm{~s}^{-1}\right)$ were typically of order $10^{-6}$ in our experiments (see tables 2 and 3 ), we calculate that $z_{\text {crit }} \approx 0.10 \mathrm{~m}$ in our experiments (equation 5.26). This implies that $z_{\text {crit }}<L_{s}$ in most of the experiments listed in tables 2 and 3 .

The estimated value of $\beta$ and equation 5.30 are used to calculate how the ratio $z_{r} / L_{s}$ changes as a function of $z_{\text {crit }} / L_{s}$. In figure $11 \mathrm{~b}$, the prediction of the model (solid line) is compared with the results of the experiments (symbols). For each experiment, we detect the distance from the virtual source at which the time-averaged light attenuation produced by the reactive plume (red line in figure 5e) is 20 times smaller than that produced by the inert plume (black line in figure 5e). At this particular threshold, the light attenuation produced by the reactive plume is too faint to be perceived with the naked eye, but it can be captured and quantified using the image analysis technique described in section 2. At larger distances from the source, however, our experimental technique becomes less accurate as the signal-to-noise ratio of the light attenuation produced by the reactive plume decreases. For each experiment, the measured distance is scaled by the stoichiometric length scale $L_{s}$, and the result is plotted in figure $11 \mathrm{~b}$ using an open or closed circle. It is seen that our experimental results compare well with the predictions of the model, with discrepancies of order $5-10 \%$ approximately.

\subsection{Light attenuation profiles obtained in case of a fast or a slow reaction}

The ratio between the critical distance $z_{\text {crit }}$ and the stoichiometric length scale $L_{s}$ can be given as a function of the entrainment, mixing and stoichiometric time scales (see 


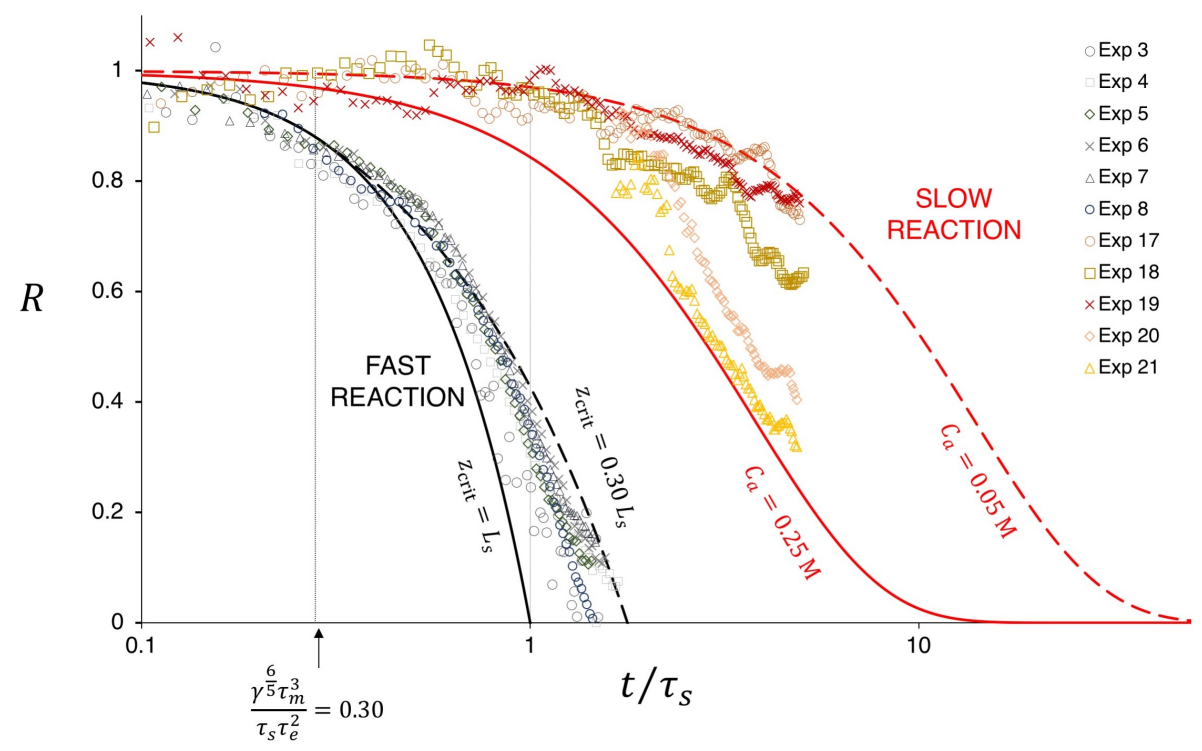

Figure 12. Fraction of the supplied chemicals which are still reactive in the plume, $R$, as a function of time. Equations 5.18 and 5.36 are used to plot the model predictions for slow- and fast-reaction experiments (red and black lines respectively). Symbols are used to illustrate the ratio betwen the time-averaged mean light attenuation produced by a reactive plume and that produced by an inert plume as measured during our experiments. On the horizontal axis, time is scaled by the stoichiometric time scale $\tau_{s}$ given by equation 5.14 .

table 5)

$$
\frac{z_{\text {crit }}}{L_{s}}=\frac{\gamma^{\frac{6}{5}} \tau_{m}^{3}}{\tau_{s} \tau_{e}^{2}}
$$

in which

$$
\gamma=\frac{\beta}{S c_{b}^{\frac{3}{2}}\left(\ln S c_{b}\right)^{\frac{9}{2}}}
$$

is used for convenience. Using equations 5.28 and 5.34, the fraction of the supplied chemicals which is still reactive at a distance $z$ below the source, $R$, is given as a function of the time $t$ required for the plume fluid to flow from the source to $z$, as

$$
R(t)= \begin{cases}1-\left(\frac{t}{\tau_{s}}\right)^{\frac{5}{3}}, & 0<\frac{t}{\tau_{s}}<\frac{\gamma^{\frac{6}{5}} \tau_{m}^{3}}{\tau_{s} \tau_{e}^{2}} \\ 1+\frac{\gamma^{2} \tau_{m}^{5}}{\tau_{s}^{\frac{5}{3}} \tau_{e}^{\frac{10}{3}}}-\frac{2 \gamma \tau_{m}^{\frac{5}{2}}}{\tau_{s}^{\frac{5}{6}} \tau_{e}^{\frac{5}{3}}}\left(\frac{t}{\tau_{s}}\right)^{\frac{5}{6}}, & \frac{\gamma^{\frac{6}{5}} \tau_{m}^{3}}{\tau_{s} \tau_{e}^{2}}<\frac{t}{\tau_{s}}<\frac{t_{r}}{\tau_{s}} .\end{cases}
$$

In equation 5.36, $t_{r}$ denotes the time required for the plume fluid to flow from the source to the level at which the instantaneous chemical reaction reaches completion, $z_{r}$. Using a combination of equations 5.30 and 5.34, we calculate that this time lapse is given by

$$
\frac{t_{r}}{\tau_{s}}=\left(\frac{\gamma^{2} \tau_{m}^{5}+\tau_{s}^{\frac{5}{3}} \tau_{e}^{\frac{10}{3}}}{2 \gamma \tau_{m}^{\frac{5}{2}} \tau_{s}^{\frac{5}{6}} \tau_{e}^{\frac{5}{3}}}\right)^{\frac{6}{5}}
$$

Equation 5.36a is used to plot the black solid curve in figure 12. This line illustrates the theoretical prediction of the minimum fraction of reactive chemicals in the plume fluid, 
$R(t)$, in case the chemical reaction is instantaneous and mixing is complete $\left(z_{\text {crit }} \geqslant L_{s}\right)$. This theoretical prediction is compared with the results of a number of fast reaction experiments (black and grey symbols, experiments 3-7 in table 2). Each symbol in the figure illustrates the ratio between the time- and depth-averaged mean intensity of light attenuation produced by a reactive plume and that produced by the associated inert plume at a given height in the tank during an experiment. It is seen that at small distances and times from the source $\left(t / \tau_{s}<0.3-0.5\right.$ and $R>0.7-0.8$ approximately), there is a relatively good agreement between the results of the experiments and the model prediction illustrated by the black solid curve. However, for $t / \tau_{s}>0.5$ and $R<0.7$ approximately, the experimental results deviate from the black solid curve: in this region, the mean concentration of reactive chemicals in the plume fluid in our experiments is larger than that predicted by equation $5.36 \mathrm{a}$ as a result of incomplete mixing. Given that $z_{\text {crit }} / L_{s}>0.30$ in all of our fast-reaction experiments (see figure $11 \mathrm{~b}$ ), we use equation $5.36 \mathrm{~b}$ to plot the dashed black curve in figure 12 , which illustrates the theoretical prediction for $R(t)$ in case $z_{\text {crit }} / L_{s}=0.30$. Figure 12 shows that most of the data points collected during the fast reaction experiments lie between the two black curves, as expected.

In figure 12, the model predictions and the results of our slow reaction experiments are plotted for comparison. For $\tau_{s} / \tau_{r} \ll 1$, we assume that the chemical species are well-mixed within the plume fluid (see section 2); this in turn implies that $R$ equals the ratio between the concentration of dye in the reactive plume fluid and that in the inert plume fluid, $C_{b \text {,reaction }} / C_{b, \text { inert }}$. We therefore use equation 5.18 to plot the solid and the dashed red curves in figure 12: these lines illustrate the two bounding profiles associated with the maximum and minimum concentrations of reactants in the ambient fluid in our experiments. It is seen that all experimental results (yellow and red symbols, experiments 17-21 in table 4) lie between these two bounding profiles as expected. Figure 12 shows that in the slow reaction experiments, a considerable amount of time is required for the consumption of the chemical species in the plume, and most of the reaction develops at times larger than $\tau_{s}$, i.e. at distances larger than $L_{s}$ below the source. Furthermore, as the concentration of the supplied chemical species in the plume fluid decreases, the rate of the pseudo-first order reaction also decreases. As indicated by equation 5.18, the pseudo-first order reaction only reaches completion when $t \rightarrow \infty$.

\section{Conclusions}

We have explored the behaviour of a reactive turbulent plume in the two limiting cases of (i) a slow reaction, which develops on a time scale longer than the time required for the entrained fluid to be mixed in the plume; and (ii) a faster reaction, which develops in a fraction of the mixing time. New experiments have been carried out using light-attenuation techniques for selected acid-base and redox chemical reactions. The measurements show that when the reaction is sufficiently slow, the entrained and source chemicals are rapidly mixed, and the reaction takes place throughout the entire volume of each eddy in the plume. However, faster reactions develop only at the periphery of each eddy, where the plume and the entrained fluids are mixed locally at the Batchelor scale (see figure $8 \mathrm{~b}$ ). The mixture of fluids in this border region becomes rapidly neutralised, while the concentration of reactants in the core of the parcel is not affected by the reaction.

Motivated by the experimental results, we have developed a model which quantifies the fraction of the entrained fluid that reacts with the source fluid in the plume. Our model indicates that the volume flux of entrained fluid increases with distance from the source 
(see equation 5.23), while the volume flux of fluid which is mixed within the plume at the Batchelor scale decreases with distance from the source (see equation 5.22). Hence, a critical level $z_{\text {crit }}$ exists at which the two fluxes are equal (see equation 5.26). In the region spanning between the plume source and this critical level, $z<z_{\text {crit }}$, the entire flux of entrained fluid is mixed locally and reacts within the plume, and so the progress of the reaction is limited by entrainment. However, at larger distances from the source, $z>z_{\text {crit }}$, only a fraction of the entrained fluid is mixed locally within the plume, and the progress of the reaction is limited by incomplete mixing. The experimental measurements of the average size of the plume eddies, $D_{i}$, and of the average size of the parcels of reactive fluid in the plume, $D_{r}$ (see figure 7 ), have enabled us to quantify $z_{\text {crit }}$ as a function of dimensionless coefficient $\beta$ (see equation 5.26 and figure 11a). As a result of the nonuniform mixing of the fluids in the plume, the distance from the source at which an instantaneous reaction is complete, $z_{r}$, is larger than that predicted using the classical plume theory, $L_{s}$. Both the longitudinal concentration profile of source chemical in the plume and the ratio $z_{r} / L_{s}$ are shown to depend on $\left(z_{\text {crit }} / L_{s}\right)^{5 / 6}$.

\section{Appendix A. Turbulent properties of the flow}

\section{A.1. Source Reynolds number, entrainment coefficient}

In each of our experiments, a turbulent, negatively-buoyant plume was formed by supplying relatively dense, dyed fluid through the nozzle located at the top of the tank (see figure 1). Based on the known source volume flux $Q_{0}$ and nozzle radius $r_{0}$ (see tables 2-4), we estimate that the mean flow speed at the nozzle was $u_{0}=0.45 \mathrm{~m} \mathrm{~s}^{-1}$ in experiments 1-16 (tables 2 and 3 ) and $u_{0}=0.88 \mathrm{~m} \mathrm{~s}^{-1}$ in experiments 17-21 (table 4). We then estimate the associated Reynolds number to be $R e_{0}=1061$ (see tables 2$4)$. It is seen that our experiments were conducted under $R e$ conditions comparable or exceeding those of many published studies on turbulent plumes, including: George et al. (1977), $R e_{0}=870 ;$ Papanicolau \& List (1988), $R e_{0}=600 ;$ Woods \& Caulfield (1992), $R e_{0} \approx 200$, here the authors note that the flow was laminar at the source, but became fully turbulent $2-3 \mathrm{~cm}$ below the source; and Ulpre et al. (2013), $R e_{0}=399$.

The time-averaged, mean velocity of the fluid descending along the plume centreline at larger distances from the source was measured in a number of experiments using the procedure described in section 2.2. Figure $3 \mathrm{c}$ shows that the measured velocities decrease with distance from the source in agreement with the theoretical prediction for fully turbulent plumes (Morton et al. 1956).

As described in section 2.2, two filling-box experiments were performed in the tank to quantify the entrainment coefficient $\alpha$ (cf. Linden et al. 1990). During these experiments, the tank was initially filled with fresh water and relatively dense, dyed inert fluid was supplied through the nozzle. The supplied fluid descended through the tank in the form of a plume, and eventually accumulated at its base. As a result, the tank became stratified, with a sharp interface separating the clear fluid at the top of the tank from the dyed fluid underneath. During each filling box experiment, we measured the rising speed of this interface, and used the collected data to estimate the volume flux in the plume as a function of distance from the source, $Q(z)$. In both experiments, we found that $Q(z) \sim z^{5 / 3}$, as predicted by the classical theory of turbulent plumes (Morton et al. 1956). We then used the measured flow rates to estimate $\alpha=0.10 \pm 0.02$ (see section 2.2 ), which is consistent with the literature for turbulent plumes. 
The results of the filling box experiments also enabled us to quantify the time scale for the experimental tank to become contaminated during an experiment. Given that the buoyancy flux $B$ in our experiments was typically of order $10^{-6} \mathrm{~m}^{4} \mathrm{~s}^{-3}$ (see tables 2-4), and given that the height of the tank was $H=0.7 \mathrm{~m}$ (see figure 1), the maximum volume flux in the plume at base of the tank was of order $Q_{\max } \approx 5 \cdot 10^{-5} \mathrm{~m}^{3} \mathrm{~s}^{-1}$ (see equation 5.1). Since the volume of ambient fluid in the tank was of order $0.12 \mathrm{~m}^{3}$, we estimate the filling time scale to be larger than $2400 \mathrm{~s}$ ( $40 \mathrm{~min}$ ) approximately. As discussed in section 2.1 , each of our experiments typically lasted 2.5-3 minutes. We therefore conclude that the impact of environmental stratification was negligible during our experiments.

\section{A.2. Impacts of source momentum flux}

As discussed in section 2.1, the source plume fluid was supplied to the experimental tank using a peristaltic pump; hence, the source volume and momentum fluxes were finite. In order to estimate the impacts of the source conditions, for each experiment listed in tables 2-4, we calculate the Morton number $\Gamma_{0}$ (Hunt \& van den Bremer 2011)

$$
\Gamma_{0}=\frac{5 B Q_{0}^{2}}{8 \alpha \pi^{\frac{1}{2}} M_{0}^{\frac{5}{2}}}
$$

Tables 2-4 show that in our experiments $\Gamma_{0}$ was of order $10^{-2}$; this indicates that our plumes were forced at the source, and that at small distances from the source the momentum $M(z)$ was larger than that of a pure plume. As a result, the descending flow behave like a buoyant jet in the near-source region. Following Hunt \& van den Bremer (2011), we calculate the length scale $L_{M_{0}}$ for the region in which the flow was affected by the initial momentum

$$
L_{M_{0}}=M_{0}^{\frac{3}{4}} B^{-\frac{1}{2}}
$$

Tables 2-4 show that the source momentum flux $M_{0}$ only affected the flow in the first 2-3 $\mathrm{cm}$ below the nozzle, while beyond this region the flow in the $70 \mathrm{~cm}$-tall tank was governed by buoyancy. As described in section 2.2 , the virtual origin of the flow, $z_{0}$, was calculated in order to account for the impacts of the source conditions. Tables 2-4 also show that the stoichiometric length $L_{s}$ was much larger than $L_{M_{0}}$ in our experiments, indicating that our measurements of the critical distance from the source at which the chemical reaction reaches completion were not affected by the initial momentum of the flow.

\section{Appendix B. Range of chemical concentrations and buoyancy fluxes used in the experiments}

The range of conditions used in our fast-reaction experiments is given in tables 2 and 3. It is seen that a number of different experiments were conducted, using plumes with: (i) different buoyancy fluxes and (ii) different concentrations of chemicals. In a first group of fast-reaction experiments (1-10 and 13-16 in tables 2 and 3), the source buoyancy flux $B$ was fixed, while the ratio between the concentration of the chemical species in the plume and in the ambient fluid, $C_{b}^{0} / C a$, was changed systematically. Since the chemical reactions used in these experiments did not affect $B$, the plume radius, speed and density were identical in this set of experiments. Tables 2 and 3 show that the critical stoichiometric dilution $D_{s}$ ranged between 15 and 129 as a result of the different concentrations of chemical species used in the experiments (see equation 5.12); this in turn led to the stoichiometric length scale $L_{s}$ varying between 10 and $38 \mathrm{~cm}$ in different 
experiments (see equation 5.13). As discussed in section 5.5, owing to incomplete mixing of the fluid in the plume, the distance from the source at which the fast reaction reached completion was up to 1.6-1.8 times larger than $L_{s}$ in our experiments (see figure $11 \mathrm{~b}$ ), leading to $z_{r}+z_{0} \approx 60-65 \mathrm{~cm}$. The investigation of the impact of a wider range of chemical concentrations would have been difficult in the laboratory. Lower concentrations of reactants in the plume fluid would have resulted in $D_{s}<10-15$ and $L_{s}<10 \mathrm{~cm}$ (see table 2): in this case, the supplied chemical species would have been neutralised at small distances from the nozzle, where the plume flow may be somehow affected by the initial momentum and source conditions (see appendix A). Higher concentrations of supplied reactant would result in $D_{s}>130-150$, and thus the reaction would have reached completion at large distances from the source, which are incompatible with the vertical dimension of the experimental tank.

Equations 5.21 and 5.26 indicate that there is one key parameter in the model, $z_{\text {crit }}$, which varies as a function of the buoyancy flux, and is not affected by the concentration of chemical species in the plume. Hence, two additional experiments (11 and 12 in table 2) were performed using the same chemical properties as in experiment 4 , but different buoyancy fluxes. In order to obtain different values of $B$, either different densities of the plume fluid, $g_{0}^{\prime}$, or different volumetric flow rates, $Q_{0}$, could have been used at the plume source. For the source conditions of experiments 11 and 12 to be as close as possible to those of the other experiments, we chose to only let $g_{0}^{\prime}$ vary, and kept the source volume flux $Q_{0}$ fixed (see table 2). This resulted in $R e_{0}, \Gamma_{0}$ and $L_{M_{0}}$ being the same across the different experiments. In order to increase the source density of the plume fluid, inert $\mathrm{NaCl}$ was added to the fluid as discussed in section 3.1. The ratio between the largest to the smallest values of $g_{0}^{\prime}$ that we could achieve was 4 . Since $z_{\text {crit }} \sim B^{-1 / 2}$ (see equations 5.21 and 5.26), this resulted in the critical distance $z_{\text {crit }}$ varying by a factor 2 among the different experiments. The impacts of such a variation on the profile of concentration of chemical species in the plume were large enough that they could be measured using our experimental procedure.

\section{REFERENCES}

Atkins, P. W. 1978 Physical Chemistry. Oxford University Press.

BAtchelor, G. K. 1959 Small-scale variation of convected quantities like temperature in turbulent fluid. J. Fluid Mech. 5, 113-133.

Bower, D. J., Caulfield, C. P., Fitzgerald, S. D. \& Woods, A. W. 2008 Transient ventilation dynamics following a change in strength of a point source of heat. J. Fluid Mech. 614, 15-37.

Campbell, A. N. \& Cardoso, S. S. 2010 Turbulent plumes with internal generation of buoyancy by chemical reaction. J. Fluid Mech. 655, 122-151.

Cardoso, S. S. \& McHugh, S. T. 2010 Turbulent plumes with heterogeneous chemical reaction on the surface of small buoyant droplets. J. Fluid Mech. 642, 49-77.

Caulfield, C. P. \& Woods, A. W. 1995 Plumes with non-monotonic mixing behaviour. Geophys. Astrophys. Fluid Dyn. 79, 173-199.

Conroy, D. T. \& Llewellyn Smith, S. G. 2008 Endothermic and exothermic chemically reacting plumes. J. Fluid Mech. 612, 291-310.

Domingos, M. G. \& Cardoso, S. S. 2013 Turbulent two-phase plumes with bubble-size reduction owing to dissolution or chemical reaction. J. Fluid Mech. 716, 120-136.

Domingos, M. G. \& CARdoso, S. S. 2015 Turbulent thermals with chemical reaction. J. Fluid Mech. 784, 5-29.

Fox, R. O. 2003 Computational Models for Turbulent Reacting Flows. Cambridge University Press. 
George, W. K., Alpert, R. L. \& Tamanini, F. 1977 Turbulence measurements in an axisymmetric buoyant plume. Int. J. Heat Mass Transfer 20, 1145-1154.

Housecroft, C. E. \& Constable, E. C. 2002 Chemistry, Equilibria. Prentice Hall, Essex.

Hunt, G. R. \& Kaye, N. G. 2001 Virtual origin correction for lazy turbulent plumes. J. Fluid Mech. 435, 377-396.

Hunt, G. R. \& van Den Bremer, T. S. 2011 Classical plume theory: 1937-2010 and beyond. IMA Journal of Applied Mathematics 76, 424-448.

Komori, S. \& UedA, H. 1984 Turbulent effects on the chemical reaction for a jet in a nonturbulent stream and for a plume in a grid-generated turbulence. Phys. Fluids 27, $77-86$.

Kundu, P. K., Cohen, I. M. \& Dowling, D. R. 2015 Fluid Mechanics. Amsterdam Academic Press.

LEAIST, D. G. 1988 The effects of aggregation, counterion binding, and added nacl on diffusion of aqueous methylene blue. Canadian Journal of Chemistry 66(9), 2452-2457.

Linden, P. F., Lane-Serff, G. F. \& Smeed, D. A. 1990 Emtying filling boxes: the fluid mechanics of natural ventilation. J. Fluid Mech. 212, 309-335.

Lupton, J. E., Delaney, J. R., Johnson, H. P. \& Tivey, M. K. 1985 Entrainment and vertical transport of deep-ocean water by buoyant hydrothermal plumes. Nature 316, $621-623$.

Mingotti, N. \& Woods, A. W. 2015 On the transport of heavy particles through a downward displacement-ventilated space. J. Fluid Mech. 774, 192-223.

Mingotti, N. \& Woods, A. W. 2016 On turbulent particle fountains. J. Fluid Mech. 793, $\mathrm{R} 1-1-\mathrm{R} 1-12$.

Morton, B. R., Taylor, G. \& Turner, J. S. 1956 Turbulent gravitational convection from maintained and instantaneous sources. Proc. R. Soc. Lond. A 234, 1-23.

Mowry, S. \& OGRen, P. J. 1999 Kinetics of methylene blue reduction by ascorbic acid. J. Chem. Educ. 76, 970-973.

Noulty, R. A. \& Leaist, D. G. 1984 Activity coefficients and diffusion coefficients of dilute aqueous solutions of lithium, sodium, and potassium hydroxides. Journal of Solution Chemistry 13, 767-778.

Papanicolau, P. N. \& List, E. J. 1988 Investigations of round vertical turbulent buoyant jets. J. Fluid Mech. 195, 341-391.

Perry, R. H. \& Green, D. W. 2008 Perry's Chemical Engineers' Handbook, Eighth Edition. McGraw-Hill.

Pope, S. B. 2000 Turbulent flows. Cambridge University Press.

Shamim, M. \& BAKI, S. M. A. 1980 Diffusion measurements in aqueous l-ascorbic acid solutions. Aust. J. Chem. 33, 1857-1861.

Snehalatha, T., Rajanna, K. C. \& Saiprakash, P. K. 1997 Methylene blue - ascorbic acid, an undergraduate experiment in kinetics. J. Chem. Educ. 74, 228-233.

Someya, S., Yoshida, S., TAватA, T. \& Oкамото, K. 2009 The effect of chemical reaction on the mixing flow between aqueous solutions of acetic acid and ammonia. International Journal of Heat and Mass Transfer 52, 4236-4243.

Sparks, R. S. J., Bursik, M. I., Carey, S. N., Gilbert, J. S., Glaze, L. S., Sigurdsson, H. \& Woods, A. W. 1997 Volcanic plumes. Wiley.

Ulpre, H., Eames, I. \& Greig, A. 2013 Turbulent acidic jets and plumes injected into an alkaline environment. J. Fluid Mech. 734, 253-274.

Vitagliano, V. \& Lyons, P. A. 1956 Diffusion in aqueous acetic acid solutions. J. Am. Chem. Soc. 78(18), 4538-4542.

Wittke, G. 1983 Reactions of phenolphthalein at various ph values. J. Chem. Educ. 60, 239240.

Woods, A. W. 2010 Turbulent plumes in nature. Annu. Rev. Fluid Mech. 42, 391-412.

Woods, A. W. \& Caulfield, C. P. 1992 A laboratory study of explosive volcanic eruptions. Journal of Geophysical Research 97, 6699-6712.

YeH, H. \& Wills, G. B. 1971 Diffusion coefficient of aqueous nitric acid at $25^{\circ} \mathrm{C}$ as function of concentration from 0.1 to 1.0 M. Journal of Chemical and Engineering Data 16, 76-77. 